\title{
Radii, masses, and ages of 18 bright stars using interferometry and new estimations of exoplanetary parameters
}

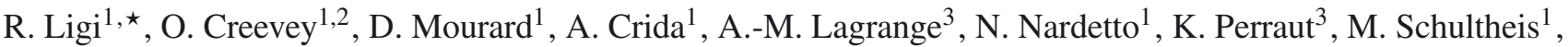 \\ I. Tallon-Bosc ${ }^{4}$, and T. ten Brummelaar ${ }^{5}$ \\ ${ }^{1}$ Laboratoire Lagrange, Université Côte d'Azur, Observatoire de la Côte d'Azur, CNRS, Boulevard de l'Observatoire, CS 34229 , \\ 06304 Nice Cedex 4, France \\ e-mail: roxanne.ligi@oca.eu \\ 2 Institut d'Astrophysique Spatiale, CNRS, UMR 8617, Université Paris XI, Bâtiment 121, 91405 Orsay Cedex, France \\ 3 Univ. Grenoble Alpes, IPAG; CNRS, IPAG, 38000 Grenoble, France \\ ${ }^{4}$ UCBL/CNRS CRAL, 9 avenue Charles André, 69561 Saint-Genis-Laval Cedex, France \\ 5 Georgia State University, PO Box 3969, Atlanta GA 30302-3969, USA
}

Received 24 July 2015 / Accepted 16 October 2015

\section{ABSTRACT}

\begin{abstract}
Context. Accurate stellar parameters are needed in numerous domains of astrophysics. The position of stars on the HertzsprungRussell diagram is an important indication of their structure and evolution, and it helps improve stellar models. Furthermore, the age and mass of stars hosting planets are required elements for studying exoplanetary systems.

Aims. We aim at determining accurate parameters of a set of 18 bright exoplanet host and potential host stars from interferometric measurements, photometry, and stellar models.

Methods. Using the VEGA/CHARA interferometer operating in the visible domain, we measured the angular diameters of 18 stars, ten of which host exoplanets. We combined them with their distances to estimate their radii. We used photometry to derive their bolometric flux and, then, their effective temperature and luminosity to place them on the H-R diagram. We then used the PARSEC models to derive their best fit ages and masses, with error bars derived from Monte Carlo calculations.

Results. Our interferometric measurements lead to an average of $1.9 \%$ uncertainty on angular diameters and 3\% on stellar radii. There is good agreement between measured and indirect estimations of angular diameters (either from SED fitting or from surface brightness relations) for main sequence (MS) stars, but not as good for more evolved stars. For each star, we provide a likelihood map in the mass-age plane; typically, two distinct sets of solutions appear (an old and a young age). The errors on the ages and masses that we provide account for the metallicity uncertainties, which are often neglected by other works. From measurements of its radius and density, we also provide the mass of $55 \mathrm{Cnc}$ independently of models. From the stellar masses, we provide new estimates of semimajor axes and minimum masses of exoplanets with reliable uncertainties. We also derive the radius, density, and mass of 55 Cnc e, a super-Earth that transits its stellar host. Our exoplanetary parameters reflect the known population of exoplanets.

Conclusions. This work illustrates how precise interferometric measurements of angular diameters and detailled modeling allow fundamental parameters of exoplanet host stars to be constrained at a level permiting analysis of the planet's parameters.
\end{abstract}

Key words. stars: fundamental parameters - planetary systems - techniques: interferometric - methods: numerical

\section{Introduction}

Stellar parameters are essential for understanding stellar physics and evolution, and they are applied to many domains of astrophysics. Several parameters are not directly measurable for single stars, such as the mass and age, and are thus derived from evolutionary models. These models are constrained by direct measurements that come from photometry, spectroscopy, or asteroseismology, for example. In particular, the radius is a fundamental parameter that takes part in the determination of many other parameters. Torres et al. (2010) highlight that the accuracy on stellar mass and radius should reach $1-3 \%$ to constrain stellar models. Directly determining the radius together with seismic parameters brings strong constraints on ages $(<10 \%$, see, e.g., Lebreton \& Goupil 2014) and stellar masses (Creevey et al. 2007). It also removes the degeneracy brought by stellar models on derived parameters. For example, Guillot \& Havel (2011)

^ Now at Aix Marseille Université, CNRS, LAM (Laboratoire d'Astrophysique de Marseille), UMR 7326, 13388 Marseille, France. calculated the parameters of the star CoRoT- 2 without any direct measurement of the radius. This parameter thus becomes a model output, in the same way as for the mass and age.

One of the most accurate ways to obtain stellar radii is to measure the angular diameter of stars (whose distance is known) using interferometry. Indeed, this method provides a direct measurement that reaches $1-2 \%$ precision on the angular diameter. The radius is then determined in a way that is as independent of models as possible. Knowing the distance and bolometric flux allows an inference of the stellar luminosity and effective temperature with little dependence on stellar atmospheres. These two quantities place stars on the Hertzsprung-Russell (H-R) diagram. Then, the generally accepted method used to determine the mass and age is to interpolate stellar evolutionary models or isochrones according to this location on the diagram. Also, stellar models and their improvement are based on the accurate location of stars on the H-R diagram. Moreover, age and mass determination of stars for which the radius is known is more reliable than only using photometry. It constitutes an important 
resource for benchmark stars, which are also used, for example, to study age, rotation, atmospheres, and activity relations, and to determine stellar gravities.

Fundamental parameters of stars are also essential for studying exoplanets. Indeed, the two main methods dedicated to exoplanet search are (i) the radial velocity (RV) method, which provides the mass function $\left(m_{\mathrm{p}} \sin (i)\right)^{3} /\left(M_{\star}+m_{\mathrm{p}}\right)^{2}$, and by supposing $m_{\mathrm{p}} \ll M_{\star}$, it gives the ratio $m_{\mathrm{p}} \sin (i) / M_{\star}$; and (ii) the transit method, which provides the ratio of the planetary and stellar radii $R_{\mathrm{p}} / R_{\star}$ and the mean stellar density. Combining this density and RV data yields the surface gravity of the planet. Transit measurements and RV are known with very good accuracy, which is significantly better than the accuracy on stellar parameters. Thus, to better constrain exoplanetary parameters, better constraints on stellar parameters are needed.

Unlike previous spatial missions, such as CoRoT and Kepler which observed faint stars, the future will be marked by the discovery and characterization of exoplanets around bright stars, meaning those accessible with interferometry. For example, CHEOPS (Broeg et al. 2013) will characterize exoplanets around already known stars brighter than $m_{V}=13$, and TESS (Ricker et al. 2014) will also discover transiting exoplanets around $4<$ $m_{V}<12$ stars. The PLATO 2.0 mission (Rauer \& Catala 2012) will search for exoplanets around bright $\left(4<m_{V}<11\right)$ stars and characterize the host stars using asteroseismology. However, scientific exploitation of these missions will be greatly reinforced by independent measurements from the ground that allow the host star to be characterized. In particular, we will get a modelindependent measure of the radius along with asteroseismic data, thus some parameters do not need to be fixed from stellar models, as for the mixing-length parameter that can then be calibrated. Furthermore, such measurements allow building very useful surface brightness (SB) relations.

When the number of angular diameters measured with interferometry increases, SB relations can be constrained with precisions on angular diameters of a few percent (1-2\%, see e.g. Kervella et al. 2004; Challouf et al. 2014; Boyajian et al. 2012). These relations still have to be improved to increase their precision (for example with homogeneous photometric measurements, in particular in the infrared domain) and accuracy (considering the stellar class for instance). Indeed, they are seldom calibrated for giants and supergiants or stars that do not have solar metallicity. However, more and more exoplanets are found around these stars.

In this paper, we present an interferometric survey of 18 stars dedicated to the measurement of their angular diameters. Section 2 describes the interferometric observations performed with VEGA/CHARA, and in Sect. 3, we explain how we derived the stellar parameters (limb-darkened diameter, radius, bolometric flux, effective temperature, metallicity, and gravity) from direct measurements. In Sect. 4, we explain how we estimate the mass and age of exoplanet host stars and discuss the results in Sect. 4.3. Finally, using our estimations of stellar masses, we present new values of minimum masses of planets and also a new estimation of the parameters of the transiting super-Earth $55 \mathrm{Cnc}$ e in Sect. 5.

\section{From visibilities to angular sizes}

We performed interferometric measurements from 2010 to 2013 using the VEGA/CHARA instrument with three telescopes at medium spectral resolution and at observing wavelengths generally between 650 and $730 \mathrm{~nm}$. We observed a spread of F, G, or K stars, including exoplanet hosts (see Table 1). The selection
Table 1. Properties of the stars of the studied sample.

\begin{tabular}{lcccc}
\hline \hline HD & Name & Sp. class & $m_{V}$ & $m_{K}$ \\
\hline 3651 & 54 Psc & K0V & 5.88 & 4.00 \\
9826 & $v$ And & F8V & 4.09 & 2.86 \\
19994 & 94 Cet & F8V & 5.07 & 3.75 \\
75732 & 55 Cnc & G8V & 5.96 & 4.05 \\
$167042^{\dagger}$ & - & K1IV & 5.97 & 3.58 \\
170693 & 42 Dra & K1.5III & 4.82 & 2.09 \\
173416 & & G8III & 6.04 & 3.85 \\
185395 & $\theta$ Cyg & F4V & 4.49 & 3.54 \\
190360 & - & G6IV & 5.73 & 4.11 \\
217014 & 51 Peg & G2IV & 5.45 & 3.91 \\
221345 & 14 And & K0III & 5.23 & 2.33 \\
\hline 1367 & $\rho$ And & K0II & 6.18 & 4.23 \\
1671 & - & F5III & 5.15 & 4.07 \\
154633 & - & G5V & 6.11 & 3.93 \\
161178 & - & G9III & 5.87 & 3.66 \\
168151 & - & K5V & 4.99 & 3.94 \\
209369 & 16 Cep & F5V & 5.03 & 3.96 \\
218560 & - & K0III & 6.21 & 3.70 \\
\hline
\end{tabular}

Notes. The eleven first ones (above the line) are known exoplanet hosts (except $\theta$ Cyg, see Ligi et al. 2012a). Photometry is given in the Johnson system (see Sect. 2). (†) HD 167042 was classified as a K1III giant by HIPPARCos, but Sato et al. (2008) classify it as a less evolved subgiant because of its position on the H-R digram.

of the host stars is described by Ligi et al. (2012b). In summary, we chose main sequence (MS), subgiant, and giant stars from the Extrasolar Planets Encyclopedia ${ }^{1}$ and sorted them according to their observability with VEGA (in term of coordinates and magnitude). We then selected stars with an expected accuracy on the angular diameter of less than $2 \%$, that is with expected angular diameters between 0.3 and 3 milliarcseconds (mas). In total, ten exoplanet host stars were observed. The first four stars observed for this project (14 And, $v$ And, 42 Dra, and $\theta$ Cyg) are presented by Ligi et al. (2012a, hereafter Paper I). Since the method used here to derive the stellar parameters differs from the one used in Paper I, we also include these stars in the present study. We also selected seven stars not known to host exoplanets and used the same criteria. The visibility curves are shown in Fig. B.1 for exoplanet host stars (except for the first four stars, see Paper I) and in Fig. B.2 for the other stars.

VEGA (Mourard et al. 2009; Ligi et al. 2013) is a spectrointerferometer working at visible wavelengths $([450-850] \mathrm{nm})$ at medium (6000) or high (30000) spectral resolution. It takes advantage of the CHARA (ten Brummelaar et al. 2005) baselines, which range from 34 to $331 \mathrm{~m}$, to reach a maximum spatial resolution of $\sim 0.3$ mas. VEGA is able to recombine the light coming from two to four of the six one-meter telescopes hosted by the CHARA array. The telescopes are arranged in a Y shape, which allows a wide range of orientations and thus a rich $(u, v)$ coverage.

Interferometry is a high angular resolution technique that measures the Fourier transform of the brightness distribution of a source, called the complex visibility. Its argument, the visibility, depends on the source size. The simplest representation of a star is the uniform disk (UD), where its intensity is considered uniform over the stellar disk. In this case, the corresponding squared visibility $\left(V^{2}\right)$ can be written as

$V^{2}(x)=\left|\frac{2 J_{1}(x)}{x}\right|^{2}$

exoplanet.eu 
Table 2. Properties of the calibrators used for VEGA observations, from the SearchCal catalog.

\begin{tabular}{llccc}
\hline \hline$\#$ & Star & $m_{V}$ & $m_{K}$ & $\theta_{\mathrm{UD}, R}$ \\
\hline 1 & HD 560 & 5.535 & 5.700 & $0.185 \pm 0.013$ \\
2 & HD 14191 & 5.571 & 5.390 & $0.251 \pm 0.018$ \\
3 & HD 10982 & 5.852 & 5.891 & $0.180 \pm 0.013$ \\
4 & HD 7804 & 5.137 & 4.921 & $0.322 \pm 0.023$ \\
5 & HD 12573 & 5.423 & 4.995 & $0.339 \pm 0.024$ \\
6 & HD 21790 & 4.727 & 4.886 & $0.270 \pm 0.019$ \\
7 & HD 88960 & 5.485 & 5.387 & $0.246 \pm 0.018$ \\
8 & HD 54801 & 5.747 & 5.366 & $0.268 \pm 0.019$ \\
9 & HD 177003 & 5.376 & 5.895 & $0.130 \pm 0.009$ \\
10 & HD 196740 & 5.051 & 5.401 & $0.176 \pm 0.012$ \\
11 & HD 190993 & 5.068 & 5.566 & $0.158 \pm 0.011$ \\
12 & HD 204414 & 5.382 & 5.290 & $0.250 \pm 0.018$ \\
13 & HD 13869 & 5.245 & 5.228 & $0.247 \pm 0.018$ \\
14 & HD 10390 & 5.629 & 5.777 & $0.175 \pm 0.012$ \\
15 & HD 214680 & 4.882 & 5.498 & $0.150 \pm 0.011$ \\
16 & HD 149212 & 4.959 & 4.961 & $0.278 \pm 0.020$ \\
17 & HD 145454 & 5.436 & 5.431 & $0.227 \pm 0.016$ \\
18 & HD 149681 & 5.552 & 4.989 & $0.353 \pm 0.025$ \\
19 & HD 204770 & 5.408 & 5.602 & $0.168 \pm 0.012$ \\
20 & HD 219485 & 5.882 & 5.872 & $0.175 \pm 0.012$ \\
\hline
\end{tabular}

Notes. Angular diameters are expressed in mas, and the error is given for the limb-darkened diameter. Magnitudes correspond to Johnson filters (see Sect. 2).

where $J_{1}(x)$ represents the first-order Bessel function and

$x=\frac{\pi B \theta_{\mathrm{UD}}}{\lambda}$,

where $B$ is the projected baseline length, $\lambda$ the observing wavelength, and $\theta_{\text {UD }}$ the UD diameter (in rad) (see, for instance, Ligi et al. 2015).

Each measurement was calibrated by stars found in the SearchCal tool ${ }^{2}$ (Bonneau et al. 2006) and followed the sequence $C-T-C$ ( $C$ referring to the calibrator and $T$ to the science target), several times in a row when possible. Calibrators were chosen by excluding variable stars and stars in multiple systems, and we used their estimated spectrophotometric UD diameter in the $R$ band (Johnson-Cousin system) to calibrate the raw squared visibility of the targets. We chose stars ideally unresolved by VEGA, i.e. whose $V^{2}$ is close to unity, which allows an accurate measurement of the transfer function of the instrument. The properties of the calibrators are given in Table 2, and we label them by a number to clarify the observing sequences given in the journal of observations (Table B.1). For example, the first observing sequence of HD 3651 used Stars $\sharp 1$ and $\sharp 2$ as calibrators. A summary of the observations is given in Table 3 . For the observing journal of $v$ And, 42 Dra, 14 And, and $\theta$ Cyg, please refer to Paper I.

From the measured visibilities, we derived $\theta_{\text {UD }}$ for each star using the LITpro software ${ }^{3}$ (Tallon-Bosc et al. 2008). These determinations are based on a large number of data points for most of the stars. Most importantly, they correspond to low $V^{2}$ and are thus very constraining for angular diameter adjustments. For the other stars, data of bad quality or difficulties getting optimal observing strategies prevented us from getting more data. The errors on angular diameters were also calculated by LITpro. To correctly account for instrumental noises in addition to the

\footnotetext{
2 Available at http://www. jmmc. fr/searchcal

3 Available at http://www.jmmc.fr/litpro_page.htm
}

Table 3. Summary of the observing journal (see Sect. 2).

\begin{tabular}{lcc}
\hline \hline Star & $\begin{array}{c}\text { Nbr. of } \\
\text { data pts. }\end{array}$ & Cal. \\
\hline HD 3651 & 26 & $1,2,3$ \\
HD 19994 & 27 & $4,5,6$ \\
HD 75732 & 20 & 7,8 \\
HD 167014 & 15 & 9 \\
HD 173416 & 10 & 10 \\
HD 190360 & 19 & $10,11,12$ \\
HD 217014 & 13 & 12,1 \\
HD 1367 & 10 & 4,5 \\
HD 1671 & 24 & $13,14,15$ \\
HD 154633 & 11 & 16,17 \\
HD 161178 & 7 & 16,18 \\
HD 168151 & 12 & 16,17 \\
HD 209369 & 11 & 19,20 \\
HD 218560 & 11 & 19 \\
\hline
\end{tabular}

photon noise and to prevent bias in the calibration process, we decided to fix the lower limit on the uncertainty on the raw squared visibility measurement to 0.05 . This method is conservative as shown by Mourard et al. $(2012,2015)$ but provides reliable uncertainties on angular diameters. We obtained an average accuracy of $1.9 \%$ on angular diameters, which is in good agreement with the requirements made when choosing the targets (see all the results in Table 4).

\section{Stellar parameters from direct measurements}

\subsection{Bolometric flux}

We determined the bolometric flux $F_{\text {bol }}$ by fitting libraries of model spectra to the literature photometry-converted-to-flux measurements. Figure 1 gives an example of the photometric energy distribution for four stars from our sample with their fits. The data were obtained using the VizieR Photometry viewer ${ }^{4}$ (data available on request). We used the BASEL empirical library of spectra (Lejeune et al. 1997), which covers a wavelength range between 9.1 and $160000 \mathrm{~nm}$. The data-fitting method incorporates a nonlinear least-squares minimization algorithm (Levenberg-Marquardt) to find the optimal scaled interpolated spectrum that fits a set of observed flux points. The method is described in detail in Creevey et al. (2015) and validated by comparing it with a second approach, originally described in van Belle et al. (2008), and using different stellar libraries. The fitting method requires on input a set of parameters $-T_{\mathrm{eff}}$, $[\mathrm{Fe} / \mathrm{H}], \log (g), A_{V}$, and a scaling factor - which define the characteristics of the spectra, the extinction to apply, and ratio of the stellar radius to the star's distance, which we denote as $\theta_{\mathrm{SED}}$ to imply it comes from the models.

We compiled the $\log (g)$ and $[\mathrm{Fe} / \mathrm{H}]$ from the literature and used the mean of these values as fixed input parameters to the fitting method. The errors in $\log (g)$ are taken as the standard deviations of the values found in the literature or a minimum of 0.1 dex. Because the stars are relatively close, the $A_{V}$ were all set to zero in a first round of analysis. However, for our subsequent analysis we adopted a value of $A_{V}$ determined using the method described in Schultheis et al. (2014). The bolometric flux is then calculated by integrating the optimal fitted spectrum. Table 5 gives the fixed input $\log (g),[\mathrm{Fe} / \mathrm{H}]$, and $A_{V}$, along with the fitted $T_{\text {eff }}$ and $\theta_{\text {SED }}$ (scaled to mas). The bolometric flux used (with $A_{V} \neq 0$ ) is given in the second-to-last column, and the

4 http://vizier.u-strasbg.fr/vizier/sed/ 
A\&A 586, A94 (2016)
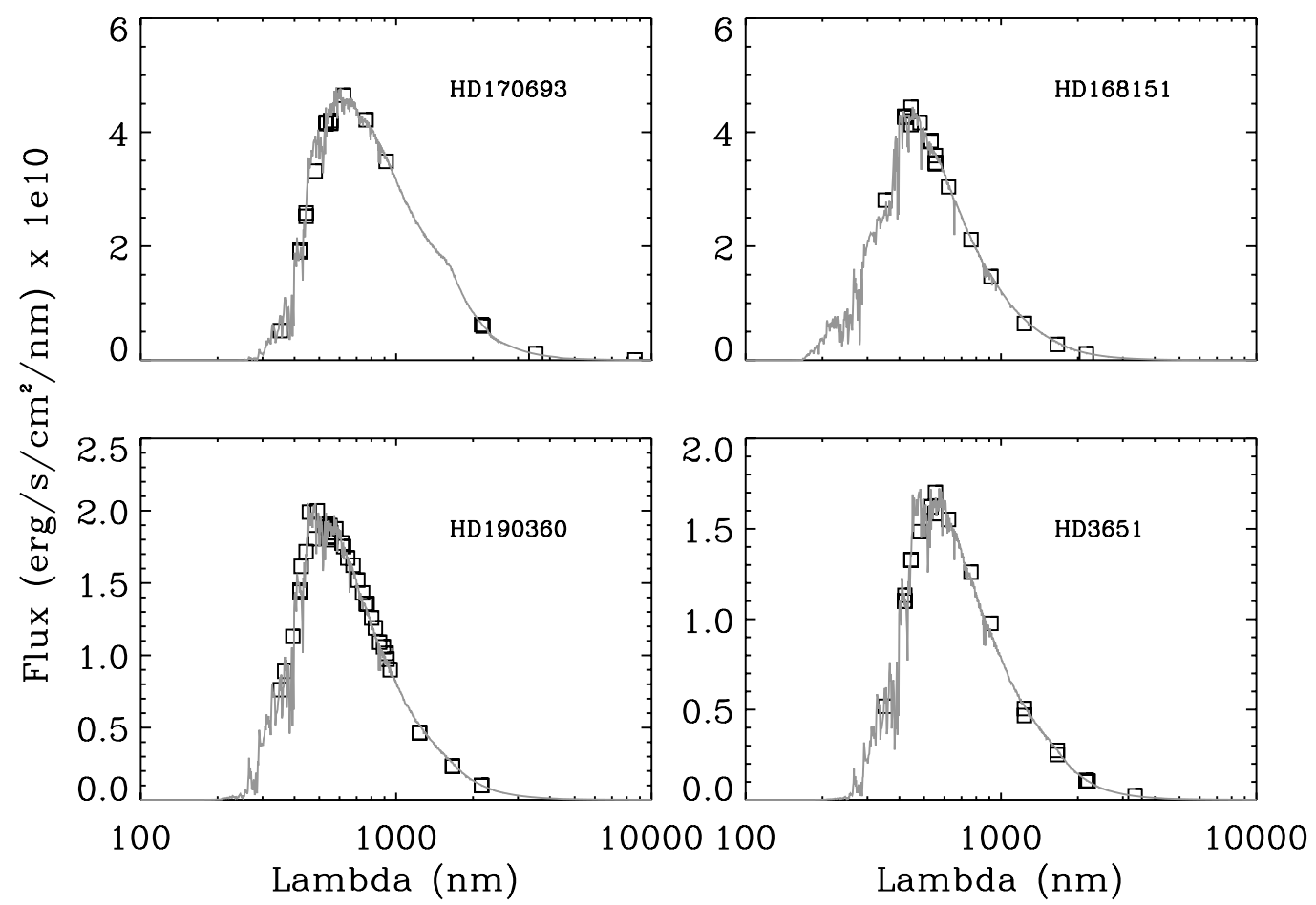

Fig. 1. Photometric energy distribution of four stars of the sample. Squares represent the photometric points and the gray curve represents the fitted spectrum (see Sect. 3.1).

Table 4. Angular diameters of our targets (in mas).

\begin{tabular}{lcccc}
\hline \hline $\mathrm{HD}$ & $\theta_{\mathrm{UD}} \pm \sigma \theta_{\mathrm{UD}}$ & $\mu_{\lambda}$ & $\theta_{\mathrm{LD}} \pm \sigma \theta_{\mathrm{LD}}(\%)$ & $\chi_{\text {red }}^{2}$ \\
\hline 3651 & $0.687 \pm 0.007$ & 0.537 & $0.722 \pm 0.007(0.97)$ & 0.97 \\
9826 & $1.119 \pm 0.026$ & 0.425 & $1.161 \pm 0.027(2.34)$ & 6.95 \\
19994 & $0.731 \pm 0.010$ & 0.448 & $0.761 \pm 0.011(1.41)$ & 0.67 \\
75732 & $0.687 \pm 0.011$ & 0.561 & $0.724 \pm 0.012(1.64)$ & 0.36 \\
167042 & $0.998 \pm 0.013$ & 0.616 & $1.056 \pm 0.014(1.28)$ & 0.30 \\
170693 & $1.965 \pm 0.009$ & 0.634 & $2.097 \pm 0.009(0.41)$ & 0.20 \\
173416 & $0.937 \pm 0.033$ & 0.608 & $0.995 \pm 0.034(3.45)$ & 0.59 \\
185395 & $0.726 \pm 0.007$ & 0.355 & $0.749 \pm 0.008(1.01)$ & 8.47 \\
190360 & $0.596 \pm 0.006$ & 0.480 & $0.622 \pm 0.007(1.08)$ & 1.00 \\
217014 & $0.624 \pm 0.013$ & 0.458 & $0.650 \pm 0.014(2.14)$ & 2.27 \\
221345 & $1.404 \pm 0.029$ & 0.614 & $1.489 \pm 0.032(2.16)$ & 2.73 \\
1367 & $0.719 \pm 0.013$ & 0.505 & $0.754 \pm 0.014(1.84)$ & 0.44 \\
1671 & $0.582 \pm 0.006$ & 0.359 & $0.600 \pm 0.006(0.92)$ & 0.42 \\
154633 & $0.763 \pm 0.011$ & 0.569 & $0.804 \pm 0.012(1.44)$ & 0.33 \\
161178 & $0.897 \pm 0.040$ & 0.545 & $0.944 \pm 0.043(4.50)$ & 1.89 \\
168151 & $0.642 \pm 0.014$ & 0.386 & $0.664 \pm 0.015(2.20)$ & 0.61 \\
209369 & $0.601 \pm 0.017$ & 0.380 & $0.621 \pm 0.018(2.85)$ & 1.72 \\
218560 & $0.875 \pm 0.020$ & 0.600 & $0.927 \pm 0.022(2.38)$ & 0.64 \\
\hline
\end{tabular}

Notes. Errors in \% are given in parenthesis (see Sect. 3.2).

bolometric flux with zero extinction is given in the last column for comparison. The errors on the fitted parameters are obtained by adding the formal error from the fit in quadrature, along with the difference in this parameter when changing the fixed parameters by $1 \sigma$, and for $[\mathrm{Fe} / \mathrm{H}]$ we used an error of 0.1 dex.

In Fig. 2, we compare the bolometric flux determined using our method assuming $A_{V}=0$ with that found in the liter-

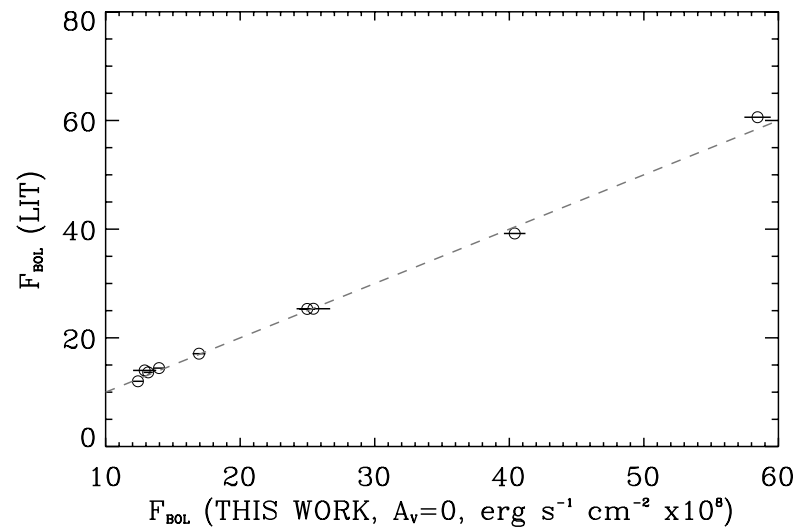

Fig. 2. Comparison between $F_{\text {bol }}$ found in the literature and $F_{\text {bol }}$ calculated in this work, for $A_{V}=0$ (see Sect. 3.1).

ature, also assuming $A_{V}=0$. The error bars are $2 \sigma$ error bars from our method (for visual purpose), showing good agreement with previous determinations. The literature values were found in van Belle \& von Braun (2009) and Boyajian et al. (2013).

\subsection{Limb-darkened diameters, effective temperatures, and radii}

Instead of UD, a more realistic representation of stars is to consider them as limb-darkened (LD) disks, which means that the intensity at the center of the stellar disk is higher than at the limb. The visibility of such objects is expressed by

$$
\begin{aligned}
V^{2}(x)= & \left(\frac{1-\mu_{\lambda}}{2}+\frac{\mu_{\lambda}}{3}\right)^{-2} \\
& \times\left[\left(1-\mu_{\lambda}\right) \frac{J_{1}(x)}{x}+\mu_{\lambda}\left(\frac{\pi}{2}\right)^{1 / 2} \frac{J_{3 / 2}(x)}{x^{3 / 2}}\right]^{2},
\end{aligned}
$$


R. Ligi et al.: Radii, masses, and ages of 18 bright stars using interferometry and new estimations of exoplanetary parameters

Table 5. Fixed input parameters to determine the bolometric flux.

\begin{tabular}{|c|c|c|c|c|c|c|c|}
\hline \multirow[b]{2}{*}{ HD } & \multicolumn{3}{|c|}{ Fixed parameters } & \multicolumn{2}{|c|}{ Fitted parameters } & \multicolumn{2}{|c|}{ Calculated parameters } \\
\hline & $A_{V}$ & {$[\mathrm{Fe} / \mathrm{H}]$} & $\begin{array}{l}\log (g) \\
{\left[\mathrm{cm} \mathrm{s}^{2}\right]} \\
\end{array}$ & $\begin{array}{l}T_{\text {eff }} \\
{[\mathrm{K}]}\end{array}$ & $\begin{array}{c}\theta_{\mathrm{SED}} \\
{[\mathrm{mas}]}\end{array}$ & $F_{\text {bol }}$ & $\begin{array}{c}F_{\text {bol }} \\
\left(A_{V}=0\right)\end{array}$ \\
\hline 3651 & 0.060 & 0.1 & $4.4 \pm 0.17$ & $5297 \pm 27$ & $0.715 \pm 0.014$ & $13.409 \pm 0.236$ & $13.163 \pm 0.169$ \\
\hline 9826 & 0.185 & 0.1 & $4.2 \pm 0.14$ & $6494 \pm 39$ & $1.073 \pm 0.016$ & $68.200 \pm 2.310$ & $58.448 \pm 0.493$ \\
\hline 19994 & 0.090 & 0.2 & $4.2 \pm 0.14$ & $6039 \pm 26$ & $0.767 \pm 0.011$ & $25.798 \pm 0.654$ & $24.980 \pm 0.291$ \\
\hline 75732 & 0.0075 & 0.3 & $4.4 \pm 0.12$ & $5219 \pm 26$ & $0.709 \pm 0.012$ & $12.435 \pm 0.168$ & $12.399 \pm 0.168$ \\
\hline 167042 & 0.103 & -0.1 & $3.2 \pm 0.10$ & $4774 \pm 33$ & $0.958 \pm 0.028$ & $15.886 \pm 0.551$ & $12.927 \pm 0.429$ \\
\hline 170693 & 0.052 & -0.5 & $2.1 \pm 0.54$ & $4460 \pm 24$ & $1.933 \pm 0.023$ & $49.180 \pm 0.600$ & $49.723 \pm 0.102$ \\
\hline 173416 & 0.047 & -0.2 & $2.5 \pm 0.10$ & $4735 \pm 23$ & $0.917 \pm 0.013$ & $13.179 \pm 0.265$ & $13.733 \pm 0.148$ \\
\hline 185395 & 0.328 & 0.0 & $4.3 \pm 0.15$ & $7181 \pm 28$ & $0.775 \pm 0.010$ & $49.400 \pm 0.460$ & $40.372 \pm 0.403$ \\
\hline 190360 & 0.044 & 0.2 & $4.3 \pm 0.09$ & $5577 \pm 26$ & $0.669 \pm 0.011$ & $14.405 \pm 0.195$ & $13.987 \pm 0.213$ \\
\hline 217014 & 0.078 & 0.2 & $4.3 \pm 0.11$ & $5804 \pm 27$ & $0.689 \pm 0.011$ & $17.965 \pm 0.238$ & $16.939 \pm 0.241$ \\
\hline 221345 & 0.046 & -0.3 & $2.4 \pm 0.29$ & $4692 \pm 25$ & $1.359 \pm 0.023$ & $27.983 \pm 0.447$ & $27.055 \pm 0.418$ \\
\hline 1367 & 0.588 & 0.0 & $3.0 \pm 0.10$ & $5488 \pm 23$ & $0.725 \pm 0.009$ & $15.959 \pm 0.432$ & $9.750 \pm 0.060$ \\
\hline 1671 & 0.473 & -0.1 & $3.7 \pm 0.10$ & $7047 \pm 27$ & $0.619 \pm 0.007$ & $31.473 \pm 0.259$ & $21.401 \pm 0.185$ \\
\hline 154633 & 0.046 & -0.1 & $3.0 \pm 0.10$ & $4934 \pm 24$ & $0.788 \pm 0.010$ & $12.243 \pm 0.211$ & $11.937 \pm 0.087$ \\
\hline 161178 & 0.408 & -0.2 & $2.4 \pm 0.25$ & $5158 \pm 26$ & $0.885 \pm 0.018$ & $19.799 \pm 0.343$ & $15.748 \pm 0.078$ \\
\hline 168151 & 0.129 & -0.3 & $4.1 \pm 0.50$ & $6563 \pm 38$ & $0.679 \pm 0.016$ & $28.519 \pm 0.674$ & $25.442 \pm 0.625$ \\
\hline 209369 & 0.116 & -0.2 & $3.8 \pm 0.10$ & $6447 \pm 41$ & $0.682 \pm 0.017$ & $26.737 \pm 0.686$ & $24.166 \pm 0.560$ \\
\hline 218560 & 0.059 & 0 & $1.5 \pm 0.10$ & $4631 \pm 24$ & $0.929 \pm 0.014$ & $13.375 \pm 0.138$ & $12.800 \pm 0.134$ \\
\hline
\end{tabular}

Notes. $F_{\text {bol }}$ is expressed in $10^{8} \mathrm{erg} \mathrm{s}^{-1} \mathrm{~cm}^{-2}$, and the error adopted in the rest of the study on $[\mathrm{Fe} / \mathrm{H}]$ is $0.1 \mathrm{dex}$. We adopt a minimum of 0.1 dex for the error in $\log (g)$ (see Sect. 3.1).

where $J_{i}$ represents the Bessel function at $i$ th order, and $\mu_{\lambda}$ the limb-darkening (LD) coefficient that defines the LD diameter $\theta_{\mathrm{LD}}$. We used the LD coefficients $\mu_{\lambda}$ from Claret \& Bloemen (2011), which depend on the metallicity $[\mathrm{Fe} / \mathrm{H}]$, gravity $\log (g)$, effective temperature $T_{\mathrm{eff}, \star}$ of the star, and the considered wavelength. Since we measure very accurate uniform angular diameters, the estimation of the limb-darkening has to be very precise as well, which requires a detailed estimation of the LD coefficients. The coefficients are given by steps of $250 \mathrm{~K}$ on $T_{\text {eff }}, 0.5$ on $\log (g)$ (where $g$ is in $\mathrm{cm} / \mathrm{s}^{2}$ ), and non-uniform steps in $[\mathrm{Fe} / \mathrm{H}]$. Since we observed around $720 \mathrm{~nm}$, we had to consider both $R$ and $I$ filters (in the Johnson-Cousin system).

We first computed linear interpolations over the coefficients corresponding to $[\mathrm{Fe} / \mathrm{H}]$ and $\log (g)$ surrounding the stellar parameters for each filter $R$ and $I$ and each temperature surrounding the initial photometric temperature (determined from $F_{\text {bol }}$ ) by $\pm 250 \mathrm{~K}$. (We took the closest values to our stars available on the tables.) Then, we averaged the resulting LD coefficients on the filters to have one coefficient per temperature. Finally, we computed linear interpolations until the derived $\theta_{\mathrm{LD}}$ calculated with the LD coefficient converge with the values of $T_{\text {eff, } \star}$ and $F_{\text {bol }}$. The final interferometric parameters are given in Table 4. We used the final LD coefficient to estimate the final $\theta_{\mathrm{LD}}$ using the LITpro software. Then, the final $T_{\mathrm{eff}, \star}$ is directly derived from the LD diameter and $F_{\text {bol }}$ :

$T_{\mathrm{eff}, \star}=\left(\frac{4 \times F_{\mathrm{bol}}}{\sigma_{\mathrm{SB}} \theta_{\mathrm{LD}}^{2}}\right)^{0.25}$,

where $\sigma_{\mathrm{SB}}$ is the Stefan-Boltzmann constant.

The stellar radius is obtained by combining the LD diameter and the distance $d$ (from HIPPARCos parallaxes, van Leeuwen 2007):

$R_{\star_{\left[R_{\oplus}\right]}}=\frac{\theta_{\mathrm{LD}_{[\mathrm{mas}]}} \times d_{[\mathrm{pc}]}}{9.305}$.
To determine the errors on $T_{\text {eff, } \star}$ and $R_{\star}$, we consider that the parameters on the righthand side of each equation are independent random variables with Gaussian probability density functions. For any quantity $X$, the uncertainty on its estimate is noted $\sigma_{X}$, and the relative uncertainty $\sigma_{X} / X$ is noted $\tilde{\sigma}_{X}$. Then, the standard deviation of each parameter that we want to estimate is given analytically to first order by a classical propagation of errors, following the formula:

$$
\begin{aligned}
& \tilde{\sigma}_{T_{\mathrm{eff}, \star}}=\sqrt{\left((1 / 2) \times \tilde{\sigma}_{\theta_{\mathrm{LD}}}\right)^{2}+\left((1 / 4) \times \tilde{\sigma}_{F_{\mathrm{bol}}}\right)^{2}} \\
& \tilde{\sigma}_{R_{\star}}=\sqrt{\tilde{\sigma}_{\theta_{\mathrm{LD}}}^{2}+\tilde{\sigma}_{d}^{2}}
\end{aligned}
$$

where $\sigma \theta_{\mathrm{LD}}, \sigma F_{\text {bol }}$, and $\sigma d$ are the errors on the LD diameter, bolometrix flux, and distance, respectively. Then, we calculate the stellar luminosity $L_{\star}$ by combining the bolometric flux and the distance :

$L_{\star}=4 \pi d^{2} F_{\mathrm{bol}}$,

and its error

$\tilde{\sigma}_{L_{\star}}=\sqrt{\left(2 \times \tilde{\sigma}_{d}\right)^{2}+\tilde{\sigma}_{F_{\mathrm{bol}}}^{2}}$.

Finally, we calculate the gravitational mass $M_{\text {grav }, \star}$ using $\log (g)$ and $R_{\star}$

$M_{\text {grav }, \star}=\frac{R_{\star}^{2} \times 10^{\log (g)}}{G}$

and its error

$\tilde{\sigma}_{M_{\mathrm{grav}, \star}}=\sqrt{\left(2 \times \tilde{\sigma}_{R_{\star}}\right)^{2}+\left(\sigma_{\log (g)} \times \ln (10)\right)^{2}}$.

The parameters and their errors are shown in Table 6. 
Table 6. Stellar parameters calculated from interferometric measurements and photometry.

\begin{tabular}{|c|c|c|c|c|c|}
\hline HD & $\begin{array}{c}T_{\mathrm{eff}, \star} \pm \sigma T_{\mathrm{eff}, \star} \\
{[\mathrm{K}]}\end{array}$ & $\begin{array}{c}d \pm \sigma d(\%) \\
{[\mathrm{pc}]}\end{array}$ & $\begin{array}{c}R_{\star} \pm \sigma R_{\star}(\%) \\
{\left[R_{\odot}\right]} \\
\end{array}$ & $\begin{array}{c}L_{\star} \pm \sigma L_{\star} \\
{\left[L_{\odot}\right]} \\
\end{array}$ & $\begin{array}{c}M_{\text {grav }, \star} \pm \sigma M_{\text {grav }, \star} \\
{\left[M_{\odot}\right]} \\
\end{array}$ \\
\hline 3651 & $5270 \pm 34$ & $11.06 \pm 0.04(0.35)$ & $0.8584 \pm 0.0088$ & $0.5103 \pm 0.0097$ & $0.68 \pm 0.26$ \\
\hline 9826 & $6243 \pm 74$ & $13.49 \pm 0.03(0.26)$ & $1.684 \pm 0.040(2.36)$ & $3.866 \pm 0.030$ & $1.64 \pm 0.53$ \\
\hline 19994 & $6063 \pm 45$ & $22.58 \pm 0.14(0.63)$ & $1.847 \pm 0.028(1.54)$ & $4.138 \pm 0.066$ & $1.97 \pm 0.64$ \\
\hline 75732 & $5165 \pm 46$ & $12.34 \pm 0.11(0.93)$ & $0.960 \pm 0.018(1.89)$ & $0.589 \pm 0.014$ & $0.85 \pm 0.24$ \\
\hline 167042 & $4547 \pm 49$ & $50.23 \pm 0.66(1.31)$ & $5.70 \pm 0.10(1.83)$ & $12.47 \pm 0.54$ & $1.88 \pm 0.07$ \\
\hline 170693 & $4280 \pm 16$ & $96.53 \pm 1.86(1.93)$ & $21.76 \pm 0.43(1.97)$ & $142.55 \pm 5.77$ & $2.17 \pm 2.70$ \\
\hline 173416 & $4544 \pm 79$ & $139.5 \pm 5.5(3.91)$ & $14.92 \pm 0.78(5.21)$ & $85.17 \pm 6.72$ & $2.57 \pm 0.27$ \\
\hline 185395 & $7305 \pm 42$ & $18.34 \pm 0.05(0.28)$ & $1.475 \pm 0.015(1.05)$ & $5.564 \pm 0.065$ & $1.58 \pm 0.55$ \\
\hline 190360 & $5781 \pm 37$ & $15.86 \pm 0.09(0.54)$ & $1.061 \pm 0.013(1.21)$ & $1.127 \pm 0.019$ & $0.82 \pm 0.17$ \\
\hline 217014 & $5978 \pm 67$ & $15.61 \pm 0.09(0.59)$ & $1.090 \pm 0.024(2.22)$ & $1.362 \pm 0.024$ & $0.86 \pm 0.22$ \\
\hline 221345 & $4483 \pm 50$ & $79.18 \pm 1.69(2.14)$ & $12.67 \pm 0.39(3.04)$ & $58.18 \pm 2.55$ & $1.47 \pm 0.99$ \\
\hline 1367 & $5382 \pm 51$ & $117.4 \pm 5.8(4.93)$ & $9.51 \pm 0.50(5.26)$ & $68.14 \pm 6.74$ & $3.30 \pm 0.35$ \\
\hline 1671 & $7133 \pm 36$ & $48.54 \pm 0.49(1.02)$ & $3.151 \pm 0.043(1.37)$ & $23.07 \pm 0.51$ & $1.82 \pm 0.05$ \\
\hline 154633 & $4883 \pm 36$ & $115.7 \pm 3.5(3.01)$ & $10.00 \pm 0.33(3.34)$ & $51.02 \pm 3.09$ & $3.65 \pm 0.24$ \\
\hline 161178 & $4992 \pm 115$ & $111.2 \pm 3.1(2.78)$ & $11.29 \pm 0.60(5.29)$ & $71.03 \pm 4.16$ & $1.17 \pm 0.68$ \\
\hline 168151 & $6638 \pm 83$ & $22.92 \pm 0.09(0.39)$ & $1.635 \pm 0.037(2.23)$ & $4.66 \pm 0.12$ & $1.23 \pm 0.15$ \\
\hline 209369 & $6755 \pm 106$ & $36.72 \pm 0.23(0.62)$ & $2.45 \pm 0.072(2.92)$ & $11.22 \pm 0.32$ & $1.38 \pm 0.08$ \\
\hline 218560 & $4637 \pm 56$ & $324.7 \pm 33.7(10.39)$ & $32.39 \pm 3.45(10.66)$ & $434 \pm 90$ & $1.21 \pm 0.26$ \\
\hline
\end{tabular}

Notes. Errors in \% are given in parenthesis (see Sect. 3.2).

\subsection{General results}

Our interferometric measurements led to precise $\theta_{\mathrm{LD}}, T_{\mathrm{eff}, \star}$, and radii $(1.9 \%, 57 \mathrm{~K}$, and $3 \%$ average accuracies with medians of $1.8 \%, 1.1 \%$, and $2.2 \%$, respectively). Some of the exoplanet host stars presented here, along with a few non-host stars, have already been observed with other long baseline interferometers (LBI), including the CLASSIC/CHARA (Sturmann et al. 2003) and the Palomar Testbed Interferometer (PTI, Colavita et al. 1999). In Fig. 3 (upper panel), we compare our estimation of $\theta_{\mathrm{LD}}$ with previous interferometric measurements. The LD diameter is achromatic (unlike the UD diameter) and should ideally be identical when estimated from different measurements and instruments. However, in practice, the estimations are not always similar, so many measurements are needed to find consistent values. Here, our values of $\theta_{\mathrm{LD}}$ are in very good agreement with previous estimations: the average relative difference $\left(\theta_{\mathrm{LD}}-\theta_{\mathrm{LBI}}\right) / \theta_{\mathrm{LBI}}$ is $-0.03 \pm 0.08\left(\theta_{\mathrm{LBI}}\right.$ being the interferometric angular diameter measured with another instrument than VEGA), and the median is -0.04 . These differences might be explained by the calibrators used for the observations or the method used to determine the LD coefficients. However, using the visible domain allows the stars to be better resolved compared to the infrared one, thus the angular diameters are better constrained. The values measured here are also in good agreement with the ones given in Paper I. The angular diameters are slightly smaller in the present analysis, but they fit within the error bars, which are also a bit larger here. The differences come from the method used to derive the LD coefficients since we kept the same data, and these coefficients are important when we reach high precisions.

We also compared our measurements with other indirect estimations of angular diameters (Fig. 3, bottom panel), including angular diameters directly determined from $F_{\text {bol }}\left(\theta_{\mathrm{SED}}\right)$ and from an empirical law determined by Kervella et al. (2004). These laws are based on photometry and calibrated by interferometry, and they allow predictions of angular diameters of dwarfs and subgiant stars. We chose to use the $V$ and $K$ bands and the associated relation $\log \left(\theta_{\mathrm{LD}}\right)=0.0755\left(m_{V}-m_{K}\right)+0.5170-0.2 m_{K}$. We consider an error of $2 \%$ in the final $\theta_{\mathrm{LD}}$, while the best estimation is $1 \%$ in their paper. The apparent magnitudes are expressed in the Johnson system, thus we had to keep the same system to calculate the angular diameters. We found the photometry of our stars in the JSDC catalog (Bonneau et al. 2006). While all the $V$ magnitudes have already been expressed in this system, for some stars, the $K$ band comes from the 2MASS reference. Thus, we converted the 2MASS magnitudes $m_{K \mathrm{~s}}$ into the Johnson system magnitudes $m_{K}$ using $m_{K}=m_{K \mathrm{~s}}+0.032^{5}$. They are given in Table 1. Again, we found good agreement between our measurements and the empirically determined angular diameters. This agreement is slightly better between angular diameters of dwarfs and subgiants determined with the Kervella et al. (2004) relation and interferometric measurements, which is expected since this law is calibrated specifically for Class V and IV stars. In both comparisons, small angular diameters measured with VEGA are generally smaller, and large angular diameters $\left(\theta_{\mathrm{LD}}>0.9\right.$ mas $)$ are a bit larger than previous values.

Concerning the effective temperatures, the average difference between $T_{\text {eff }}$ (directly determined from SED fitting) and $T_{\text {eff, } \star}$ (from interferometric measurements) $\left(T_{\text {eff, } \star}-T_{\text {eff }}\right) / T_{\text {eff }}$ is $-0.007 \pm 0.030$. This is shown in Fig. 4. It is not surprising to find such differences between the temperatures since they are correlated with the angular diameters and have been obtained with the same $F_{\text {bol }}$. As for angular diameters, the largest discrepancies are found for stars not on the MS: the relative difference is on average $-0.023 \pm 0.022$ for giants and bright giants, whereas it is of $0.003 \pm 0.030$ for dwarfs and subgiants. Our estimations of temperatures are slightly lower than the estimations from SED for low temperatures and slightly higher for high temperatures.

\section{Ages and masses of stars}

Once a star is placed on the H-R diagram, we determine its mass and age using stellar evolution models. This is crucial to providing benchmark stars to stellar physicists and also to better understanding planetary systems. In this section, we perform this for the 18 stars of our sample with careful attention given to determining the errors.

\footnotetext{
http://www . pas. rochester. edu/ emamajek/ memo_BB88_Johnson2MASS_VK_K.txt
} 
R. Ligi et al.: Radii, masses, and ages of 18 bright stars using interferometry and new estimations of exoplanetary parameters
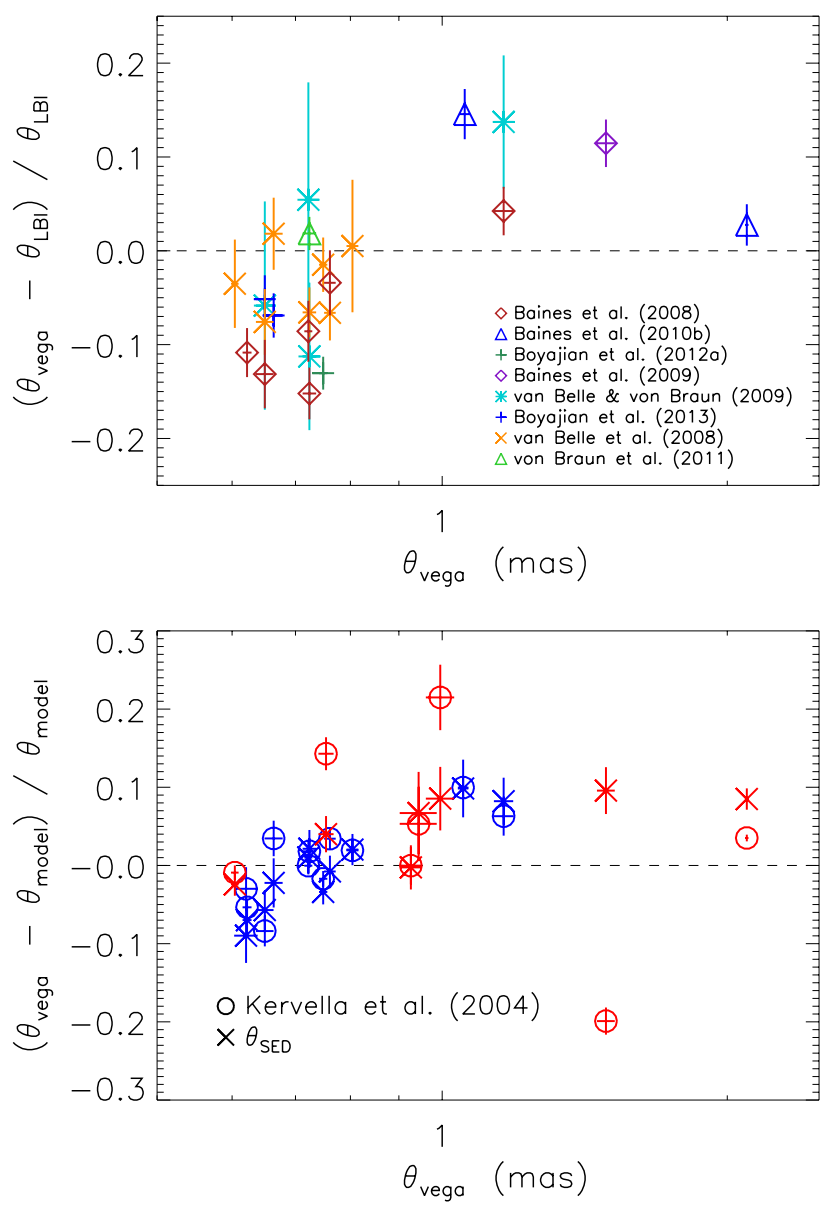

Fig. 3. Upper panel: comparison between angular diameters measured with VEGA and with other instruments. Bottom panel: estimation of empirically determined angular diameters versus angular diameters measured with VEGA. Dwarfs and subgiants stars are plotted in blue, and giants and bright giants in red (see Sect. 3.3).

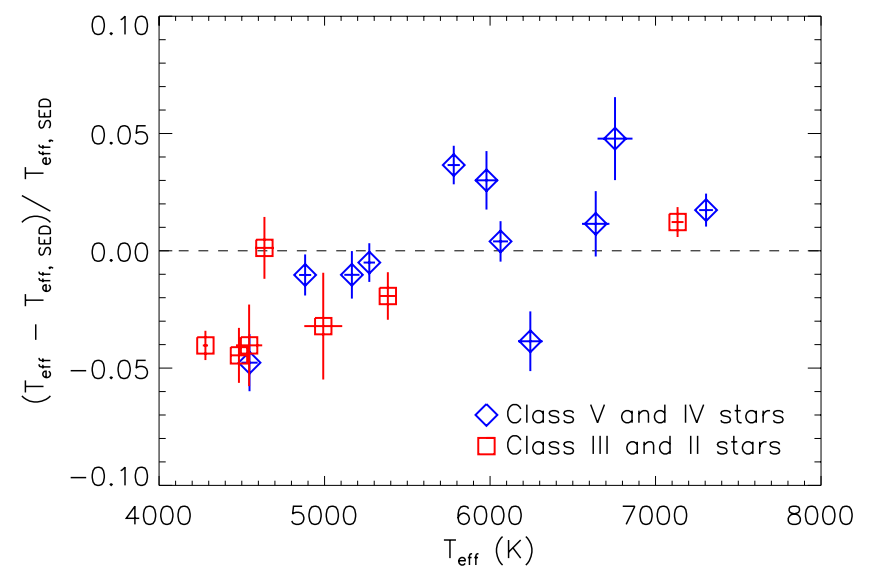

Fig. 4. Comparison between interferometric temperatures and temperatures derived from SED. Dwarfs and subgiants stars are plotted with blue diamonds, and giants and bright giants with red squares (see Sect. 3.3).

We used the recently published PARSEC stellar models (Bressan et al. 2012) to determine the masses and ages of the 18 stars. The details of these models are well documented in Bressan et al. (2012), but here we give a brief summary. Models are initiated on the pre-main sequence phase and evolve beyond the horizontal branch, which is sufficient for our purposes. High temperature opacity tables (OPAL, Iglesias \& Rogers 1996) are used in conjunction with those calculated from their own code (Aesopus, Marigo \& Aringer 2009) for lower temperatures. The models make use of the FREEEOS $\operatorname{code}^{6}$ to calculate the equation of state, and the nuclear reaction rates comprise the $\mathrm{p}-\mathrm{p}$, $\mathrm{Ne}-\mathrm{Na}$, and $\mathrm{Mg}-\mathrm{Al}$ chains, the $\mathrm{CNO}$ cycle, and some alphacapture reactions.

Energy transport in the convective regions is described by the mixing-length theory of Böhm-Vitense (1958), and the mixinglength parameter found for the Sun is 1.74. Convective overshoot from the convective core and below the convective envelope is a variable parameter that depends on stellar mass and chemical composition. Microscopic diffusion is included following the implementation of Salasnich (1999). The reference distribution of heavy elements is given by Grevesse \& Sauval (1998) except for some species where the Caffau et al. (2011) ones are used, and this gives a present solar metallicity of $Z_{\odot}=0.01524$ and $Z_{\odot} / X_{\odot}=0.0207$. A chemical enrichment law is derived from the solar value using the primordial helium abundance $(0.2485)$, and this is given as $Y=0.2485+1.78 Z$. The approximation $[\mathrm{M} / \mathrm{H}]=\log \left(Z / Z_{\odot}\right)$ is used to determine the metallicity.

The isochrones span log(age) from 6.6 to 10.13 in steps of 0.01 and $[\mathrm{M} / \mathrm{H}]$ from 0.5 to $-0.8 \mathrm{dex}$ in steps of $\sim 0.015$. We assume that $[\mathrm{M} / \mathrm{H}]=[\mathrm{Fe} / \mathrm{H}]$ because no additional information is available to differentiate them.

For this data to be appropriate, the points on one single isochrone should not be separated on the H-R diagram by a large distance compared to $\sigma_{L_{\star}}$ and $\sigma_{T_{\mathrm{eff}, \star}}$. As this is generally not the case, we performed spline interpolations of each isochrone to produce a refined table for each star around $L_{\star}$ and $T_{\star}$, except for HD 1367 and HD 218560 due to their complex position on the H-R diagram. For these two stars, we did not build any interpolation, which gives more consistent results.

\subsection{Best fit (least squares)}

To find the mass and age of a star, we perform a least squares algorithm, looking for the parameter combination in our table that minimizes the quantity:

$\chi^{2}=\frac{\left(L-L_{\star}\right)^{2}}{\sigma_{L_{\star}}{ }^{2}}+\frac{\left(T_{\mathrm{eff}}-T_{\mathrm{eff}, \star}\right)^{2}}{\sigma_{T_{\mathrm{eff}, \star}}{ }^{2}}+\frac{\left([\mathrm{M} / \mathrm{H}]-[\mathrm{M} / \mathrm{H}]_{\star}\right)}{\sigma_{[\mathrm{M} / \mathrm{H}]_{\star}}{ }^{2}}$.

Although not intrinsically degenerate (because the number of constraints equals that of parameters to be determined given a fixed set of parameters), this problem does not have a unique solution, especially in some parts of the H-R diagram, where the isochrones cross, so that a given luminosity and temperature may correspond to two stars of different ages and masses. Typically, there is a young ( $<400 \mathrm{Myr})$ and an old (>400 Myr) solution. This is described particularly well by Bonfanti et al. (2015, Fig. 2), who also show that two solutions are possible when also using the PARSEC tables, one in the Gyr range and the other in the Myr range. They show that without knowledge of the stellar mass, it is not possible to establish the evolutionary stage of the star. Additional stellar properties may allow one to rule out one of the two solutions (e.g. chromospheric activity, Lithium abundance, gyrochronology, or independent measure of the stellar mass, see discussion about HD 75732 below), but we

6 http://freeeos.sourceforge.net/ 
Table 7. Masses and ages derived from PARSEC isochrones (see Sect. 4).

\begin{tabular}{|c|c|c|c|c|c|c|c|c|}
\hline \multirow[t]{2}{*}{ Star } & \multicolumn{4}{|c|}{ Old solution } & \multicolumn{4}{|c|}{ Young solution } \\
\hline & $\begin{array}{c}M_{\star} \pm \sigma(\%) \\
{\left[M_{\odot}\right]}\end{array}$ & $\begin{array}{c}\text { Age } \pm \sigma(\%) \\
{[\mathrm{Gyr}]}\end{array}$ & $\begin{array}{c}\log (g) \\
{\left[\mathrm{cm} \mathrm{s}^{2}\right]}\end{array}$ & $\chi^{2}$ & $\begin{array}{c}M_{\star} \pm \sigma(\%) \\
{\left[M_{\odot}\right]}\end{array}$ & $\begin{array}{c}\text { Age } \pm \sigma(\%) \\
{[\mathrm{Myr}]}\end{array}$ & $\begin{array}{l}\log (g) \\
{\left[\mathrm{cm} \mathrm{s}^{2}\right]}\end{array}$ & $\chi^{2}$ \\
\hline HD 3651 & $0.848 \pm 0.040$ & $10.00 \pm 3.52(35)$ & 4.6 & 0.0009 & $0.889 \pm 0.024$ & $38.90 \pm 3.686(9)$ & 3.2 & 0.027 \\
\hline HD 9826 & $1.312 \pm 0.075(5.72)$ & $3.02 \pm 0.92(30)$ & 4.2 & 0.0008 & $1.359 \pm 0.027(1.99)$ & $14.79 \pm 1.446(10)$ & 1.3 & 0.020 \\
\hline HD 19994 & $1.317 \pm 0.079(5.96)$ & $3.80 \pm 0.84(22)$ & 4.8 & 0.0064 & $1.459 \pm 0.023(1.58)$ & $12.30 \pm 1.326(11)$ & 4.3 & 0.0095 \\
\hline HD 75732 & $0.874 \pm 0.013$ & $13.19 \pm 1.18(9)$ & 4.3 & 0.919 & $0.968 \pm 0.018$ & $30.90 \pm 3.028(10)$ & 4.3 & 0.0124 \\
\hline HD 167042 & $1.646 \pm 0.311(18.88)$ & $1.82 \pm 5.23(287)$ & 4.3 & 7.766 & - & - & - & - \\
\hline HD 170693 & $0.879 \pm 0.050(7.86)$ & $13.19 \pm 1.92(15)$ & 4.7 & 2.309 & - & - & - & - \\
\hline HD 173416 & $1.336 \pm 0.258$ & $3.47 \pm 3.18(92)$ & 3.2 & 0.0003 & - & - & - & - \\
\hline HD 185395 & $1.49 \pm 0.060(4.01)$ & $0.50 \pm 0.40(79)$ & 4.0 & 0.0002 & $1.519 \pm 0.037(2.42)$ & $18.62 \pm 4.436(24)$ & 4.4 & 0.013 \\
\hline HD 190360 & $1.04 \pm 0.059(5.71)$ & $4.79 \pm 2.51(52)$ & 4.6 & 0.0011 & $1.073 \pm 0.045(4.22)$ & $29.51 \pm 2.11(7)$ & 4.2 & 0.049 \\
\hline HD 217014 & $1.12 \pm 0.063(5.66)$ & $1.996 \pm 2.24(112)$ & 1.5 & $\sim 0.0$ & $1.138 \pm 0.073(6.39)$ & $27.54 \pm 0.177(6)$ & 3.0 & 0.019 \\
\hline HD 221345 & $0.898 \pm 0.069(7.63)$ & $13.19 \pm 2.10(16)$ & 4.3 & 0.1569 & - & - & - & - \\
\hline HD 1367 & $2.560 \pm 0.089(3.46)$ & $0.575 \pm 0.05(8)$ & 2.9 & 0.254 & - & - & - & - \\
\hline HD 1671 & $1.875 \pm 0.075(4.00)$ & $1.203 \pm 0.14$ & 0.3 & 0.1834 & $2.074 \pm 0.037(1.78)$ & $4.47 \pm 0.341(8)$ & 0.4 & 0.0091 \\
\hline HD 154633 & $1.962 \pm 0.207(10.5)$ & $1.175 \pm 0.52(45)$ & 3.1 & 0.0051 & - & - & - & - \\
\hline HD 161178 & $1.936 \pm 0.212(11.0)$ & $1.446 \pm 0.49$ & 0.7 & 0.0052 & - & - & - & - \\
\hline HD 168151 & $1.189 \pm 0.068$ & $3.468 \pm 0.76(22)$ & 3.8 & $\sim 0.0$ & $1.294 \pm 0.025(1.95)$ & $12.30 \pm 1.082(9)$ & 2.4 & 0.0126 \\
\hline HD 209369 & $1.531 \pm 0.061(3.99)$ & $1.95 \pm 0.24(12)$ & 3.5 & 0.0067 & $1.666 \pm 0.033(1.96)$ & $6.918 \pm 0.708(10)$ & 4.3 & 0.0002 \\
\hline HD 218560 & $3.638 \pm 0.441(12.13)$ & $0.263 \pm 0.12(44)$ & 2.0 & 0.0225 & - & - & - & - \\
\hline
\end{tabular}

choose to report both here for completeness, in contrast to many authors who keep only one solution without justification ${ }^{7}$.

The two solutions are given in Table 7, with the uncertainties computed in next section. The value of $\chi^{2}$ is provided, and generally $\ll 1$, showing very good agreement. If $\chi^{2}$ is large, it means that no model corresponds to this luminosity, temperature, and metallicity within the acceptable uncertainty. We discuss these particular cases in Sect. 4.3. The isochrones corresponding to the old and young ages are shown in Fig. B.3 for each star with $L_{\star}, T_{\text {eff, } \star}$ and their error bars as a red cross.

\subsection{Likelihood maps and calculation of uncertainties}

The likelihood function $\mathcal{L}$ gives the probability of getting the observed data for a given set of stellar parameters. Generally, it is easy to express it as a function of the observables, but it is tricky to express it as a function of the physical parameters one wants to determine. Here, we want the probability density function of the stellar mass and age, which is an expression of $\mathcal{L}$ as a function of $M_{\star}$ and age ${ }^{8}$, not of $L_{\star}, T_{\text {eff, } \star}$, and [M/H]. For more details on the likelihood and a Bayesian approach, the reader is referred to Pont \& Eyer (2004) and Jørgensen \& Lindegren (2005).

To produce a simplified map of $\mathcal{L}$ in the $\left(M_{\star}\right.$, age $)$ plane, we pick random points in the H-R diagram such that each of

\footnotetext{
7 That some of our stars have planets is not a suggestion to constrain their age either. These stars no longer have protoplanetary disks (inside which the planet forms, e.g., Armitage 2013), so they are older than 5 Myr (e.g., Mamajek 2009), but nothing can exclude their being as young as $10 \mathrm{Myr}$.

8 More precisely, the probability distribution function (PDF) of the mass and age is proportionnal to $\mathcal{L} \times f_{0}$, where $f_{0}$ is the prior. Assuming a constant star formation rate in the history of the Galaxy, $f_{0}$ should be independent of the age; although the IMF is not flat, our uncertainties on the masses are small enough that a flat prior in $M_{\star}$ is acceptable. In the end, $f_{0}$ can be taken uniform, and the PDF is simply proportional to $\mathcal{L}\left(M_{\star}\right.$, age $)$.
}

the three terms on the righthand side of Eq. (11) is less than 1, 2 , and 3 . And we find the corresponding mass and age by least squares, as described above, and plot them in the $\left(M_{\star}\right.$, age) plane in red, yellow, or blue. The resulting maps are displayed in Fig. B.4. These crude contour plots of the likelihood show two important features: (i) the mass and age are far from being independent, and they show a clear negative correlation in the old solution, as expected; (ii) for a majority of stars in our sample, the likelihood has two distinct peaks, and the marginal PDF of the age is far from Gaussian. These two peaks correspond to the young and old solutions discussed previously, which are the local modes of $\mathcal{L}$ in these two regions.

Maps of the joint PDF of the mass and age are the most information-rich way of presenting the results. However, they are not very convenient to use. Below, we therefore estimate the standard deviation in age and mass for each of the two solutions. We do not have a precise and detailed map of the PDF, but a Monte-Carlo (MC) method is appropriate, as shown by Jørgensen \& Lindegren (2005).

Simply drawing independent Gaussian sets of $L_{\star}$ and $T_{\mathrm{eff}}, \star$ would erase the natural correlation between $L_{\star}$ and $T_{\mathrm{eff}, \star}$, which occurs because they are both increasing functions of $F_{\text {bol }}$. (The covariance between $L_{\star}$ and $T_{\mathrm{eff}, \star}$ is proportional to the variance of $F_{\text {bol }}$, see Appendix A.) To have a more realistic and smaller cloud of points, we proceed as follows:

1. draw 1500 quadruplets $\left\{F_{\text {bol }}, d, \theta,[M / H]\right\}$, as the occurrences of four independent Gaussian random variables of appropriate mean and standard deviation;

2. combine them into 1500 triplets:

$$
\left\{L_{\star}=4 \pi F_{\text {bol }} d^{2}, T_{\text {eff }, \star}=\left(4 F_{\text {bol }} / \sigma_{\mathrm{SB}} \theta^{2}\right)^{1 / 4},[\mathrm{M} / \mathrm{H}]\right\}
$$

3. apply the least squares procedure described above to find the corresponding $1500\left\{M_{\star}\right.$, age $\}$ pairs;

4. compute the mean and standard deviation of the masses and ages found and their correlation. 
The clouds of points are shown in Fig. B.3. In the top lefthand panel (HD 3651), the cloud of points is particularly elongated. It shows that this procedure captures the expected correlations because it starts from the most basic parameters. We stress that in the literature, error bars are usually poorly described and derived, even though they are of outmost importance for astrophysical interpretation.

As clearly explained in Pont \& Eyer (2004), the estimator of the ages and masses provided by the MC approach may be biased. Thus, we do not report the average ages and masses obtained in Table 7, but we have checked that they are very close to the best fit value in most of the cases (within a quarter of the standard deviation).

\subsection{Discussion}

The errors on masses are generally $\sim 7.6 \%$ for the old solution, but the errors on the ages are much larger. The young solutions give errors of $\sim 10 \%$ because there are generally fewer possibilities at $1 \sigma$ for younger ages.

Concerning MS stars, we always find two solutions thanks to their position on the H-R diagram (except for HD 154633), unlike for giant stars. The best fit results give $\chi^{2} \ll 1$, the ones corresponding to old solutions being smaller than the ones for young solutions. However, these quantities remain very low, which means that without additional constrains, it is difficult to choose one solution over the other, as explained in the previous section. Only for two stars (HD 170693 and HD 167042) are the results not consistent with the models $\left(\chi^{2} \gg 1\right)$. One possibility for explaining inconsistency with the models is that the mixing-length parameter in the models should be adjusted, (see, e.g., Creevey et al. 2012; Bonaca et al. 2012). One would require a downward revision of this parameter to make the models fit. Including an extra free parameter gives another degree of freedom, which clearly enlarges the uncertainties. However, with only a few constraints, one is required to use stellar models with a fixed mixing-length parameter.

The masses derived in this paper are in good agreement with the ones derived for the four stars in Paper I, except for HD 221345. The mass estimated here is more than twice lower than the first estimation, but agrees more with other values in the literature (see below). This is directly due to the $\log (g)$ used in Paper I, which is close to the one used in the present study but is not sufficient to describe a star at an evolved stage. Besides, the gravitational masses (Table 6) are not in good agreement with the masses derived from models because of the $\log (g)$ used to calculate them. Indeed, the $\log (g)$ derived from the best fit method is generally different from the one shown in Table 5, and this is particularly true for giants. In what follows, we discuss the results for each star and compare them with other previous results.

HD 167042 - When comparing angular diameters, one of the most important discrepancies is found for HD 167042. Baines et al. (2010) find $\theta_{\text {Baines }}=0.92 \pm 0.02$ mas, which gives $\theta_{\mathrm{LD}} / \theta_{\mathrm{Baines}}=1.145$. This directly affects the determination of other parameters such as the luminosity and temperature (there is a difference of $4.8 \%$ between $T_{\text {eff }}$ and $T_{\text {eff, } \star}$ ) that determine the position of the star in the H-R diagram. However, our angular diameter measurement is close to the estimation by Kervella et al. (2004). Concerning the age, no solution better than $3 \sigma$ is found, which translates into $\chi^{2}=7.8$, thus we cannot conclude anything about the true age of the star.
HD 75732/55 Cnc - The star HD 75732 is a MS star but shows a $\chi^{2}$ slightly higher than the other stars of the same spectral type. We found an angular diameter that does not agree with the Baines et al. (2008) measurement, which is much larger (0.854 mas) than the one we found. However, our angular diameter of $55 \mathrm{Cnc}$ is similar at $1.02 \%$ to the one derived by von Braun et al. (2011, $\theta=0.711 \pm 0.004$ mas), who did an extensive study of the system of $55 \mathrm{Cnc}$. Thus, we compare our results with those found by von Braun et al. (2011). When setting the values of $\theta, F_{\text {bol }}, T_{\text {eff }},[\mathrm{M} / \mathrm{H}]$ (from Valenti \& Fischer 2005), and $L_{\star}$ given in their paper as input parameters in our code, we find a mass of $0.87 M_{\odot}$ and an age of $13.2 \mathrm{Gyr}$, in agreement with our results. For the young solution, we obtain $0.96 M_{\odot}$ and 33.8 Myr, which is slightly older than our solution. The smaller errors can be explained by the lower error on the metallicity (0.04 dex), as shown in Sect. 4.4. These results are very consistent with the masses and ages we determined from the parameters derived in the present paper. However, we find an older star than that with the Yonsei-Yale model used by von Braun et al. $(2011,10.2 \pm 2.5 \mathrm{Gyr})$, and we find two solutions. It is not clear though whether the Yonsei-Yale method provides only one or two solutions.

Maxted et al. (2015) give a mean stellar density of $55 \mathrm{Cnc}$ directly from the analysis of the transit of $55 \mathrm{Cnc}$ e and Kepler's law: $\rho_{\star}=1.084_{-0.036}^{+0.040} \rho_{\odot}$. Using this value and the radius which we measured $\left(R_{\star} \stackrel{-0.036}{=} 0.96 \pm 0.02 R_{\odot}\right)$, we obtain a modelindependent stellar mass of $0.960 \pm 0.067 M_{\odot}$ (taking a mean error of $0.038 \rho_{\odot}$ on the stellar density). This mass is very consistent with the one derived from isochrones and that corresponds to the young solution $\left(0.968 \pm 0.018 M_{\odot}\right)$. Thus, this direct estimate of the mass favors the young age (31 Myr) derived from isochrones. And since this mass comes from a direct estimation, we use it to derive planetary parameters in the rest of the paper.

HD 3651/54 Psc - The PARSEC models reproduce this star well. The radius we measured is slightly larger than the one measured by van Belle $\&$ von Braun $\left(2009,0.81 R_{\odot}\right)$ but in very good agreement with the Baines et al. (2008) value $\left(0.85 R_{\odot}\right)$. From chromospheric activity, Wright et al. (2004) derived an age of $5.9 \mathrm{Gyr}$, which is much younger than our solution, but this would tempt us to adopt our old solution rather than the young one. In contrast, Boyajian et al. (2013) find an older age (14.9 Gyr) using Yonsei-Yale isochrones, but they do not take the error on the metallicity into account, and they specify that their method can lead to unrealistic age determinations, especially for the lowest luminosity stars.

HD 9826/v And - This star has been studied extensively because it hosts at least four exoplanets (Curiel et al. 2011). Its age was determined several times, as were its mass, radius, and temperature; they are fully summarized in McArthur et al. (2010). Our estimations of these parameters are in good agreement with the previous determinations. For example, the age and mass fall well within the previous estimations that are set between 2.3 and 5 Gyr for the age and 1.24 and $1.31 M_{\odot}$ for the mass, and these were determined using different methods.

HD 19994/94 Cet - Many age determinations have been performed concerning this star. Wright et al. (2004) found an age of $3.55 \mathrm{Gyr}$, in good agreement with our estimation. Using the Baines et al. (2008) radius, Boyajian et al. (2013) found an older star of $4.8 \mathrm{Gyr}$ along with a mass of $1.275 M_{\odot}$, also in good 
agreement. In the catalog of the Geneva Copenhagen Survey (GCS; Holmberg et al. 2009), the age of 3 Gyr is given for HD 19994. Saffe et al. (2005) used the chromospheric activity to determine the age of HD 19994 and found a much larger estimation (13.01 Gyr), which is also older than the other estimations made by them using isochrones $(4.7 \mathrm{Gyr})$ and lithium abundances (1.4 Gyr). The latter would favor our older solution.

HD 170693/42 Dra - We only find an old solution for HD 170693 with a high $\chi^{2}$. Bonfanti et al. (2015) used the same isochrones as we did (PARSEC tables) to characterize this star and found an age and mass of $9 \mathrm{Gyr}$ and $1.0 \pm 0.1 M_{\odot}$. However, the input parameters were not the same in their study, and we stress here that, unlike them, we bring a direct determination of the radius that is a free parameter for them and is equal to $20.9 \pm 0.6 R_{\odot}$. Our error on $T_{\mathrm{eff}, \star}$ is very small, and it is most likely this parameter that dominates our solution. A better precision on the parallax would allow verification of the very small error on the temperature.

HD 173416 - There are not many studies concerning this star. Bonfanti et al. (2015) find that it is younger than our estimation $(1.5 \pm 0.6 \mathrm{Gyr})$, and this is to our knowledge the only determination of the age before ours. They found a mass of $1.8 M_{\odot}$, which is closer to Liu et al. $\left(2009,2.0 \pm 0.3 M_{\odot}\right)$ and Liu et al. $\left(2010,2.37 M_{\odot}\right)$ estimations using Yonse-Yale isochrones. None of them used a direct angular diameter measurement as input in their model.

HD 185395/ $\theta$ Cyg - This star has long interested scientists for the unusual radial velocity variations it presents and make it a complex system not fully understood yet, suspecting several planets around the star (see Ligi et al. 2012a, and references therein for additional information). Guzik et al. (2011) discuss the solar-like oscillations it shows and the probability of having $\gamma$-Dor pulsations by considering two stellar masses, 1.38 and $1.29 M_{\odot}$ with different metallicities. They state that for solar metallicity (as we consider in our study), $\gamma$ Dor $g$-mode pulsators, expected masses are higher, on the order of 1.6 $M_{\odot}$, which is a value close to the estimation of the mass we found.

HD 190360 - A wide range of ages has been found for HD 190360; 11.3 Gyr (Boyajian et al. 2013) using the Baines et al. (2008) radius; $6.7 \mathrm{Gyr}$ along with a mass of $0.96 M_{\odot}$ (Naef et al. 2003); Ibukiyama \& Arimoto (2002) gave $12.11 \mathrm{Gyr}$; Valenti \& Fischer (2005) gave 7.2 Gyr; and Bensby et al. (2014) estimated 4.9 Gyr with an upper limit of 9.4 Gyr and a lower limit of $2.8 \mathrm{Gyr}$, along with a mass of $0.99_{-0.06}^{+0.05} M_{\odot}$, among others. This is a good example that shows how difficult the age and mass determination is and how it depends on input parameters and model. Our mass estimation is in very good agreement with the Fuhrmann $\left(1998 ; 1.04 M_{\odot}\right)$ and Bensby et al. (2014; $0.99_{-0.06}^{0.05} M_{\odot}$ ) estimations.

HD 217014/51 Peg - This star is known as the first solarlike star around which an exoplanet has been found (Mayor \& Queloz 1995). We find masses consistent with a solar type star, but younger. Our estimation of the age is closer to that of Bonfanti et al. $(2015,3.3 \pm 1.2 \mathrm{Gyr})$, in particular concerning the mass $\left(1.1 \pm 0.02 M_{\odot}\right)$. The reanalysis of the GCS data in Casagrande et al. (2011) uses two sets of models, and they find a median age and mass of $5.33 \mathrm{Gyr}$ and $1.06 M_{\odot}$, and 7.4 Gyr and $1.02 M_{\odot}$ with PADOVA and BASTI, respectively.

HD 221345/14 And - The only previous age and mass determinations we found for this star are those from Bonfanti et al. $\left(2015,3.20 \pm 2.10 \mathrm{Gyr}\right.$ and $\left.1.40 \pm 0.20 M_{\odot}\right)$ and Baines et al. $\left(2009,4.5 \pm 1.9 \mathrm{Gyr}\right.$ and $\left.1.1 \pm 0.2 M_{\odot}\right)$, and this last mass is in good agreement with our estimation. As shown previously, the estimation of these parameters is difficult, and one can find as many values as there are estimations. Baines et al. (2009) measured a smaller angular diameter than we did, which translates into a higher luminosity and might explain the difference. However, there is a strong discrepancy between empirically determined angular diameters and our measurement: we found $1.49 \pm 0.03$ mas but $\theta_{\mathrm{SED}}=1.359 \pm 0.023$ mas and $\theta_{\text {Kervella }}=1.859$ mas. This might be explained by the fact that 14 And is a giant, so the Kervella et al. (2004) relation is not appropriate for this star. Also, the infrared photometry is not homogeneous with the visible part. There also are discrepancies between $T_{\text {eff }}$ and $T_{\text {eff, }}(4.5 \%$ difference).

The comparisons for the non-host stars are more difficult since for most of them, we bring here the first estimation of their mass and age. Casagrande et al. (2011) provide an estimation of the age and mass of HD 1671, HD 168151, and HD 209369. For HD 1671, we find a slightly younger star than Casagrande et al. (2011), but the masses are consistent, particularly when comparing with those obtained with the PADOVA code (1.82 $M_{\odot}$ for both median and most probable masses). Concerning HD 168151, our age estimation is between the Boyajian et al. (2013, 5 Gyr) and Casagrande et al. (2011, $\sim 2.5 \mathrm{Gyr}$ ) results, and our mass estimation is a bit lower. Finally, Casagrande et al. (2011) give very similar results to ours for HD 209369.

\subsection{On the role of metallicity}

Taking the uncertainty on the metallicity into account significantly increases the range of the distributions of the masses and ages, id est the final error bars. To quantify the error bugdet due to the metallicity, we take the case of HD 3651 as an example, for which we have reasonable errors and low $\chi^{2}$. When setting the error on the metallicity to $\sigma([\mathrm{M} / \mathrm{H}])=0.001$ dex (instead of 0.1 dex, see Sect. 3.1), we get errors of $22 \%$ and $2.1 \%$ on the age and mass, respectively, for the old solution. Thus, the error on the metallicity contributes to one third of the total error of the age and to half of the error on the mass. It is even more significant for the young solution, where the errors reduce to $3 \%$ and $0.43 \%$ for the age and mass. If we only consider the uncertainty on the metallicity (reducing the errors on $F_{\text {bol }}, \theta$, and $d$ by a factor $10^{-5}$ ), we get very similar errors on the age and mass than the ones shown in Table 7: the errors are of $31 \%$ and $4.37 \%$ on the age and mass for the old solution, and $9 \%$ and $2.5 \%$ for the young solution. This emphasizes the significant contribution of the error on the metallicity. Standard deviations assuming a fixed metallicity are therefore underestimated and should be considered as a lower limit.

\section{Exoplanetary parameters}

\subsection{Planetary masses, semi-major axes, and habitable zone}

Radial velocity measurements constitute one of the two most prolific methods used to discover exoplanets. It gives the 
R. Ligi et al.: Radii, masses, and ages of 18 bright stars using interferometry and new estimations of exoplanetary parameters

Table 8. Orbital parameters of planets (see Sect. 5.1).

\begin{tabular}{|c|c|c|c|c|c|c|}
\hline Star & Planet & $\begin{array}{c}\omega \\
{[\mathrm{deg}]}\end{array}$ & $\begin{array}{c}P \\
\text { [days] }\end{array}$ & $\begin{array}{c}K \\
{\left[\mathrm{~m} \mathrm{~s}^{-1}\right]}\end{array}$ & $e$ & Ref. \\
\hline HD 3651 & $\mathrm{~b}$ & $233.3 \pm 7.4$ & $62.21 \pm 0.02$ & $16.0 \pm 1.2$ & $0.62 \pm 0.05$ & Butler et al. (2006) \\
\hline \multirow[t]{4}{*}{ HD 9826} & $\mathrm{~b}$ & $324.9 \pm 3.8$ & $4.617 \pm 2.3 e-5$ & $70.51 \pm 0.45$ & $0.0215 \pm 7 . e-4$ & Curiel et al. (2011) \\
\hline & $\mathrm{c}$ & $258.8 \pm 0.43$ & $1276.46 \pm 0.57$ & $68.14 \pm 0.45$ & $0.2987 \pm 0.0072$ & \\
\hline & $\mathrm{d}$ & $241.7 \pm 1.6$ & $241.26 \pm 0.06$ & $56.26 \pm 0.52$ & $0.2596 \pm 0.0079$ & \\
\hline & $\mathrm{e}$ & $367.3 \pm 2.3$ & $3848.86 \pm 0.74$ & $11.54 \pm 0.31$ & $0.00536 \pm 0.00044$ & \\
\hline HD 19994 & $\mathrm{~b}$ & $41.0 \pm 8.0$ & $535.7 \pm 3.1$ & $36.2 \pm 1.9$ & $0.300 \pm 0.040$ & Butler et al. (2006), Mayor et al. (2004) \\
\hline \multirow[t]{5}{*}{ HD 75732.} & $\mathrm{~b}$ & $110 \pm 54$ & $14.65 \pm 0.0001$ & $71.11 \pm 0.24$ & $0.004 \pm 0.003$ & Endl et al. (2012) \\
\hline & $\mathrm{c}$ & $356 \pm 22$ & $44.38 \pm 0.007$ & $10.12 \pm 0.23$ & $0.07 \pm 0.02$ & \\
\hline & $\mathrm{d}$ & $254 \pm 32$ & $4909 \pm 30$ & $45.2 \pm 0.4$ & $0.02 \pm 0.008$ & \\
\hline & $\mathrm{e}$ & $90 \pm 0$ & $0.736546 \pm 3 . e-6$ & $6.30 \pm 0.21$ & $0 . \pm 0$ & \\
\hline & $\mathrm{f}$ & $139 \pm 8$ & $261.2 \pm 0.4$ & $6.2 \pm 0.3$ & $0.32 \pm 0.05$ & \\
\hline HD 167042 & $\mathrm{~b}$ & $82 \pm 52$ & $417.6 \pm 4.5$ & $33.3 \pm 1.6$ & $0.101 \pm 0.066$ & Sato et al. (2008) \\
\hline HD 170693 & $\mathrm{~b}$ & $218.7 \pm 10.6$ & $479.1 \pm 6.2$ & $110.5 \pm 7$ & $0.38 \pm 0.06$ & Döllinger et al. (2009) \\
\hline HD 173416 & $\mathrm{~b}$ & $254 \pm 11$ & $323.6 \pm 2.2$ & $51.8 \pm 2.0$ & $0.21 \pm 0.04$ & Liu et al. (2009) \\
\hline \multirow[t]{2}{*}{ HD 190360} & $\mathrm{~b}$ & $12.4 \pm 9.3$ & $2891 \pm 85$ & $23.5 \pm 0.5$ & $0.36 \pm 0.03$ & Vogt et al. (2005) \\
\hline & $\mathrm{c}$ & $153.7 \pm 32$ & $17.10 \pm 0.015$ & $4.6 \pm 1.1$ & $0.01 \pm 0.1$ & \\
\hline HD 217014 & $\mathrm{~b}$ & $58 \pm 0$ & $4.23 \pm 3.6 e-5$ & $55.94 \pm 0.69$ & $0.013 \pm 0.012$ & Butler et al. (2006) \\
\hline HD 221345 & $\mathrm{~b}$ & $0 \pm 0$ & $185.84 \pm 0.23$ & $100.0 \pm 1.3$ & $0 \pm 0$ & Sato et al. (2008) \\
\hline
\end{tabular}

Table 9. Semi-major axes and minimum masses of exoplanets, and habitable zones of host stars derived from orbital parameters (found in the literature) and stellar parameters estimated in this work from isochrones (see Sect. 5.1).

\begin{tabular}{ll|cc|cc|c}
\hline \hline \multirow{2}{*}{ Star } & \multirow{2}{*}{ Planet } & \multicolumn{2}{|c|}{ Old solution } & \multicolumn{2}{c|}{ Young solution } & \\
& & $a$ & $m_{\mathrm{p}} \sin (i)$ & $a$ & $m_{\mathrm{p}} \sin (i)$ & $\mathrm{HZ}$ \\
& & {$[\mathrm{au}]$} & {$\left[M_{\mathrm{Jup}}\right]$} & {$[\mathrm{au}]$} & {$\left[M_{\mathrm{Jup}}\right]$} & {$[\mathrm{au}]$} \\
\hline HD 3651 & $\mathrm{b}$ & $0.2908 \pm 0.0045$ & $0.220 \pm 0.021$ & $0.2955 \pm 0.0026$ & $0.227 \pm 0.021$ & $0.62-1.23$ \\
HD 9826 & $\mathrm{b}$ & $0.0594 \pm 0.0116$ & $0.692 \pm 0.027$ & $0.06010 \pm 0.00040$ & $0.708 \pm 0.010$ & $1.56-3.14$ \\
& $\mathrm{c}^{\dagger}$ & $2.521 \pm 0.049$ & $4.16 \pm 0.16$ & $2.551 \pm 0.017$ & $4.257 \pm 0.064$ & \\
& $\mathrm{~d}$ & $0.830 \pm 0.016$ & $1.994 \pm 0.079$ & $0.8401 \pm 0.0056$ & $2.041 \pm 0.033$ & \\
& $\mathrm{e}$ & $5.26 \pm 0.10$ & $1.066 \pm 0.050$ & $5.324 \pm 0.035$ & $1.091 \pm 0.033$ & \\
HD 19994 & $\mathrm{b}$ & $1.415 \pm 0.029$ & $1.658 \pm 0.111$ & $1.4639 \pm 0.0096$ & $1.775 \pm 0.098$ & $1.64-3.29$ \\
HD 167042 & $\mathrm{b}$ & $1.281 \pm 0.089$ & $1.67 \pm 0.24$ & - & - & $3.24-6.42$ \\
HD 170693 & $\mathrm{b}$ & $1.148 \pm 0.031$ & $3.61 \pm 0.31$ & - & - & $11.15-22.14$ \\
HD 173416 & $\mathrm{b}$ & $1.016 \pm 0.066$ & $2.08 \pm 0.28$ & - & - & $8.46-16.79$ \\
HD 190360 & $\mathrm{b}$ & $4.02 \pm 0.11$ & $1.573 \pm 0.073$ & $4.067 \pm 0.098$ & $1.61 \pm 0.06$ & $0.88-1.76$ \\
& $\mathrm{c}$ & $0.1314 \pm 0.0025$ & $18.97 \pm 4.58^{*}$ & $0.1331 \pm 0.0019$ & $19.42 \pm 4.69^{*}$ & \\
HD 217014 & $\mathrm{b}$ & $0.0532 \pm 0.0010$ & $0.480 \pm 0.019$ & $0.0534 \pm 0.0011$ & $0.485 \pm 0.021$ & $0.95-1.90$ \\
HD 221345 & $\mathrm{b}$ & $0.615 \pm 0.015$ & $2.61 \pm 0.14$ & - & - & $7.02-13.9$ \\
\hline
\end{tabular}

Notes. We took $M_{\text {Jup }}=1.8986 \times 10^{27} \mathrm{~kg} .{ }^{(*)}$ Expressed in Earth mass, with $M_{\oplus}=5.9736 \times 10^{24} \mathrm{~kg} .{ }^{(\dagger)}$ Lies in the HZ.

minimum mass of the exoplanet $m_{\mathrm{p}} \sin (i)$ :

$m_{\mathrm{p}} \sin (i)=\frac{M_{\star}^{2 / 3} P^{1 / 3} K\left(1-e^{2}\right)^{1 / 2}}{(2 \pi G)^{1 / 3}}$

where $m_{\mathrm{p}}$ and $M_{\star}$ are the planetary and stellar masses, $P$ and $K$ are the period and the semi-amplitude of the radial velocity signal, $e$ is the eccentricity of the planet, and $G$ is the gravitational constant. Thus, to determine the minimum mass of the exoplanet itself, one has to know the stellar mass. In the previous section, we give the stellar masses of ten exoplanet host stars (see Table 7), which yields the semi-major axes $a$ and the masses of their exoplanets using the observables $P$ and $K$ given in Table 8. For half of the stellar sample, there are two solutions concerning the age and mass (an old one and a young one), thus we give the corresponding semi-major axes and planetary masses for each solution. Our errors on $a$ account for the uncertainty in the stellar mass (which is not always the case in the literature) derived from the MC method. The planetary parameters are given in Table 9. The old and young sets of planetary parameters are generally very close to each other, sometimes 


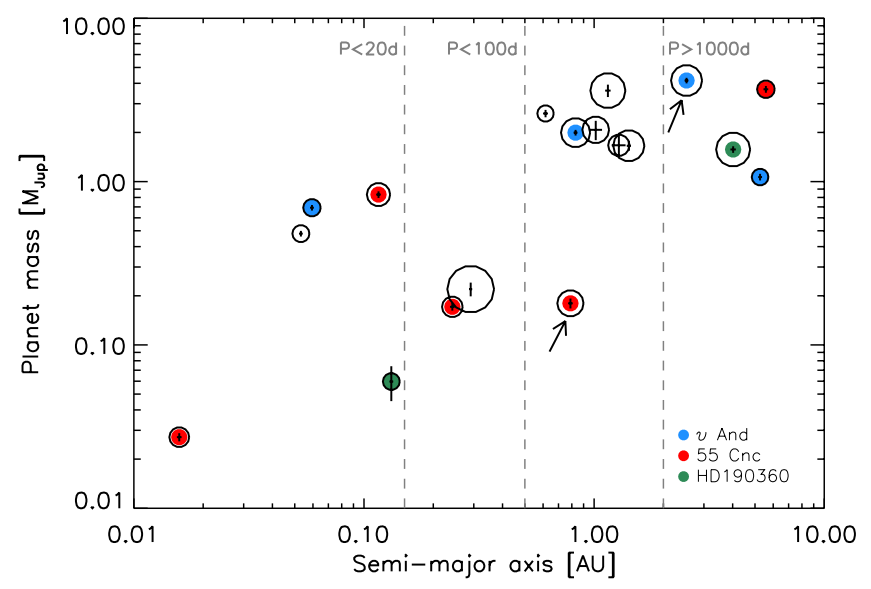

Fig. 5. Minimum masses of exoplanets versus their distance to the star from the old solution for the stellar mass (except for $55 \mathrm{Cnc}$, whose planetary minimum masses come from a direct estimate of the mass). Symbol sizes are an increasing function of the eccentricity of planets. The arrows indicate the planets in the HZ, and multiple planet systems are shown in color (see Sect. 5.1).

Table 10. Semi-major axes and minimum masses of exoplanets of the $55 \mathrm{Cnc}$ system derived from orbital parameters (found in the literature, see Table 8) and a direct estimation of the stellar mass (see Sect. 4.3).

\begin{tabular}{|c|c|c|}
\hline Planet & $\begin{array}{c}a \\
{[\mathrm{au}]}\end{array}$ & $\begin{array}{c}m_{\mathrm{p}} \sin (i) \\
{\left[M_{\mathrm{Jup}}\right]}\end{array}$ \\
\hline $\mathrm{b}$ & $0.1156 \pm 0.0027$ & $0.833 \pm 0.039$ \\
\hline $\mathrm{c}$ & $0.2420 \pm 0.0056$ & $0.1711 \pm 0.0089$ \\
\hline $\mathrm{d}$ & $5.58 \pm 0.13$ & $3.68 \pm 0.17$ \\
\hline $\mathrm{e}$ & $0.01575 \pm 0.00037$ & $8.66 \pm 0.50^{*}$ \\
\hline $\mathrm{f}^{\dagger}$ & $0.789 \pm 0.018$ & $0.180 \pm 0.012$ \\
\hline
\end{tabular}

Notes. ${ }^{(*)}$ Expressed in Earth mass, with $M_{\oplus}=5.9736 \times 10^{24} \mathrm{~kg} .{ }^{(\dagger)}$ Lies in the HZ, located between 0.67 and 1.33 au.

almost identical, because the young and old stellar masses are not dramatically different. Thus, Fig. 5 only shows the solutions derived from the old solution for the stellar masses. However, a planet of a given mass has a different structure after a few dozen Myr or a few Gyr of evolution, so the fact that a young solution exists matters. The system of $55 \mathrm{Cnc}$ does not appear in Table 9 since it has a direct determination of the mass that does not correspond to either a young or an old solution. The parameters of this system are thus given in Table 10 .

The habitable zone (HZ) is defined as a range of distances where liquid water can be found on an exoplanet. We used the method described by Jones et al. (2006) to calculate it. We first calculate the critical flux at the inner boundary

$S_{\mathrm{b}, i}\left(T_{\mathrm{eff}, \star}\right)=\left(4.190 \times 10^{-8} T_{\mathrm{eff}, \star}^{2}\right)-\left(2.139 \times 10^{-4} T_{\mathrm{eff}, \star}\right)+1.296$

and at the outer boundary

$S_{\mathrm{b}, o}\left(T_{\mathrm{eff}, \star}\right)=\left(6.190 \times 10^{-9} T_{\mathrm{eff}, \star}^{2}\right)-\left(3.319 \times 10^{-5} T_{\mathrm{eff}, \star}\right)+0.2341$, where $S_{\mathrm{b}}\left(T_{\mathrm{eff}, \star}\right)$ is given in units of the solar constant and $T_{\mathrm{eff}, \star}$ in $\mathrm{K}$. We can then calculate the inner and outer distances of the $\mathrm{HZ}$ in au:

$$
\begin{aligned}
& r_{i}=\left[\frac{L_{\star}}{S_{\mathrm{b}, i}\left(T_{\mathrm{eff}, \star}\right)}\right] \\
& r_{o}=\left[\frac{L_{\star}}{S_{\mathrm{b}, o}\left(T_{\mathrm{eff}, \star}\right)}\right],
\end{aligned}
$$

where $L_{\star}$ is the luminosity of the star in $L_{\odot}$ from Table 6 . The resulting values are given in Table 9 for each star. Jones et al. (2006) specify that this method is based on a simplified model that neglects enhanced cloud formation and the formation of $\mathrm{CO}_{2}$ clouds, which results in a conservative $\mathrm{HZ}$. Thus, the $\mathrm{HZ}$ could in reality be wider. As expected, the values of $\mathrm{HZ}$ found by Jones et al. (2006) are close to our estimations when the stellar parameter estimations are in good agreement. This is the case for HD 9826, HD 217014, and HD 19994. For HD 75732, HD 3651, and HD 190360, we found HZ to be closer to their star than what is given by Jones et al. (2006). It is the same for the planetary masses, which depend on the stellar masses and thus explain differences between different estimations. As noted in Sect. 4.3, for example, our estimation of the mass of HD 221345 is lower than what is estimated in Paper I. This directly translates into a lower minimum mass for HD $221345 \mathrm{~b}$.

According to our values, only HD $9826 \mathrm{c}$ and HD $75732 \mathrm{flie}$ in their HZ. They are large exoplanets (of the Jupiter type), thus life as we know it could hardly been found on them. However, their moons could be terrestrial bodies with water on their surface and possibly an atmosphere, if these planets have a system similar to those of the solar system giant planets (think of Titan and Europa).

In Fig. 5, we see that small exoplanets lie closer to their stellar host than large planets. This is of course due to an instrumental bias, but our sample is quite representative of the population of known exoplanets.

\subsection{The case of $55 \mathrm{Cnc} e$}

The system of $55 \mathrm{Cnc}$ holds a transiting super-Earth, $55 \mathrm{Cnc}$ e, which was independently discovered by Winn et al. (2011) and Demory et al. (2011). The transit method provides the ratio of the planetary to the stellar radius and the density of the star. Thus, to correctly determine the planetary radius $R_{\mathrm{p}}$, one has to know the stellar radius. This method also provides the inclination of the system. If RV measurements are also performed, which is the case for the system of $55 \mathrm{Cnc}$, the true planetary mass $M_{\mathrm{p}}$ can then be derived, contrary to the minimum mass that is currently found. Then, the density $\rho_{\mathrm{p}}$ of the planet can be derived. Von Braun et al. (2011) give a complete review of this system using at first interferometric measurements to determine 55 Cnc's radius. Here, we consider our interferometric measurement for the radius and our direct determination of the mass to derive 55 Cnc e's radius, mass, and density.

The results are given in Table 11. We calculated them using the transit parameters given by Dragomir et al. (2014). For the planetary mass, we do not consider the error on the inclination $i$ since it is negligible (it implies a variation on the order of $1 \%$ o on the error on the mass). Since the stellar radius and density are known, we can express the planetary density as

$\rho_{\mathrm{p}}=\frac{3^{1 / 3}}{2 \pi^{2 / 3} G^{1 / 3}} \rho_{\star}^{2 / 3} R_{\star}^{-1} T D^{-3 / 2} P^{1 / 3} K\left(1-e^{2}\right)^{1 / 2}$, 
Table 11. Parameters of 55 Cnc e derived from this work using the transit values given by Dragomir et al. (2014) (see Sect. 5.2).

\begin{tabular}{cc}
\hline \hline \multicolumn{2}{c}{$55 \mathrm{Cnc} \mathrm{e}$} \\
\hline$R_{\mathrm{p}}\left[R_{\oplus}\right]$ & $2.031_{-0.088}^{+0.091}$ \\
$M_{\mathrm{p}}\left[M_{\oplus}\right]$ & $8.631 \pm 0.495$ \\
$\rho_{\mathrm{p}}\left[\mathrm{g} \mathrm{cm}^{-3}\right]$ & $5.680_{-0.749}^{+0.709}$ \\
\hline
\end{tabular}

where TD refers to the transit depth caused by the planet. This expression of $\rho_{\mathrm{p}}$ is independent of $M_{\star}$ and directly linked to measured quantities. It therefore allows for a precise estimate of the planetary density with small uncertainties from a standard propagation of errors. The mass we find $\left(8.631 \pm 0.495 M_{\oplus}\right)$ places 55 Cnc e just below the no-iron line in Fig. 7 of Demory et al. (2011) and between the 50\% water and the Earth-like lines of Fig. 3 in Winn et al. (2011). Our results are also in good agreement with the radius and density given by Dragomir et al. (2014) and Winn et al. (2011), but are more accurate thanks to an accurate and direct determination of the stellar radius and density, since the error bar on $\rho_{\mathrm{p}}$ is dominated by the error on TD. We thus confirm that $55 \mathrm{Cnc}$ e can be classified as a super-Earth or a mini-Neptune.

These results illustrate that the knowledge of exoplanet characteristics pass through the knowledge of stellar parameters. Their accuracy are decisive in detecting exoplanets potentially hosting life.

\section{Conclusion}

We performed interferometric measurements with the VEGA/CHARA instrument in visible wavelength to measure the angular diameter of 18 stars. Our measurements are very constraining for adjustments as we reach low $V^{2}$, and we got many data points. We thus reached an average of $1.9 \%$ accuracy on angular diameters. These angular diameters are generally consistent with previous interferometric measurements or with the estimations using the Kervella et al. (2004) empirical law. However, a bigger discrepancy is found toward giant stars and stars with angular diameters larger than 1 mas. Using photometry, we derived the luminosity and effective temperatures with an average precision of $57 \mathrm{~K}$, which allowed us to place the stars in the H-R diagram. Then, we used PARSEC models to derive stellar masses and ages. To do so, we used a best fit approach and a MC approach to estimate the error bars, which give consistent results except for the few stars whose $L_{\star}$ and $T_{\mathrm{eff}, \star}$ appear to not be consistent with the models. For those stars, a better estimation of the metallicity or a different mixing-length parameter should lead to a better match with the models.

We showed that for the same luminosity and temperature, several solutions can be found, especially for MS stars. Each time, an old solution and a young solution gave ages in the Myr and the Gyr range, respectively, in agreement with Bonfanti et al. (2015). However, the masses are generally similar for the two solutions. In any case, finding the age of a star is not an easy exercise because this parameter depends on the model, and the problem is degenerate. We also showed that the error on the metallicity is very decisive and that not taking an error on the metallicity into account yields a dramatic underestimation of the errors on the age and mass.

Using orbital parameters given by radial velocity measurements and the newly determined stellar masses, we characterized the ten exoplanetary systems included in our sample; that is, we computed the minimum masses and semi-major axes of exoplanets with reliable error bars. These results reproduce the population of known exoplanets well. We also derived the habitable zone of these systems and verified that two planets lie in their HZ, although they are not Earth-like. We looked in more detail at the super-Earth $55 \mathrm{Cnc}$ e because it transits its star. We therefore gave a new estimation of its radius, mass, and density, which we find to be more precise than previous measurements.

The progression from interferometric observations of host stars to planetary parameters is complex. It should be realized with care, paying attention to the propagation of errors at each step. This is the price to pay to get accurate values with realistic uncertainties, which benefit from the direct measurement of the radius of the stars.

Acknowledgements. We acknowledge the anonymous referee, whose constructive and relevant remarks led to a significant improvement of our paper. R.L. acknowledges the support from the Observatoire de la Côte d'Azur after her Ph.D. We acknowledge all the CHARA team and the VEGA team for the interferometric observations. This research made use of The Extrasolar Planets Encyclopaedia at exoplanet.eu and of the VizieR catalog access tool, CDS, Strasbourg, France.

\section{References}

Armitage, P. J. 2013, Astrophysics of Planet Formation (Cambridge University Press)

Baines, E. K., McAlister, H. A., ten Brummelaar, T. A., et al. 2008, ApJ, 680, 728

Baines, E. K., McAlister, H. A., ten Brummelaar, T. A., et al. 2009, ApJ, 701, 154

Baines, E. K., Döllinger, M. P., Cusano, F., et al. 2010, ApJ, 710, 1365

Bensby, T., Feltzing, S., \& Oey, M. S. 2014, A\&A, 562, A71

Böhm-Vitense, E. 1958, Z. Astrophys., 46, 108

Bonaca, A., Tanner, J. D., Basu, S., et al. 2012, ApJ, 755, L12

Bonfanti, A., Ortolani, S., Piotto, G., \& Nascimbeni, V. 2015, A\&A, 575, A18

Bonneau, D., Clausse, J.-M., Delfosse, X., et al. 2006, A\&A, 456, 789

Boyajian, T. S., McAlister, H. A., van Belle, G., et al. 2012, ApJ, 746, 101

Boyajian, T. S., von Braun, K., van Belle, G., et al. 2013, ApJ, 771, 40

Bressan, A., Marigo, P., Girardi, L., et al. 2012, MNRAS, 427, 127

Broeg, C., Fortier, A., Ehrenreich, D., et al. 2013, in EPJ Web Conf., 47, 3005

Butler, R. P., Wright, J. T., Marcy, G. W., et al. 2006, ApJ, 646, 505

Caffau, E., Ludwig, H.-G., Steffen, M., Freytag, B., \& Bonifacio, P. 2011, Sol. Phys., 268, 255

Casagrande, L., Schönrich, R., Asplund, M., et al. 2011, A\&A, 530, A138

Challouf, M., Nardetto, N., Mourard, D., et al. 2014, A\&A, 570, A104

Claret, A., \& Bloemen, S. 2011, A\&A, 529, A75

Colavita, M. M., Wallace, J. K., Hines, B. E., et al. 1999, ApJ, 510, 505

Creevey, O. L., Monteiro, M. J. P. F. G., Metcalfe, T. S., et al. 2007, ApJ, 659, 616

Creevey, O. L., Thévenin, F., Boyajian, T. S., et al. 2012, A\&A, 545, A17

Creevey, O. L., Thévenin, F., Berio, P., et al. 2015, A\&A, 575, A26

Curiel, S., Cantó, J., Georgiev, L., Chávez, C. E., \& Poveda, A. 2011, A\&A, 525, A78

Demory, B.-O., Gillon, M., Deming, D., et al. 2011, A\&A, 533, A114

Döllinger, M. P., Hatzes, A. P., Pasquini, L., et al. 2009, A\&A, 499, 935

Dragomir, D., Matthews, J. M., Winn, J. N., \& Rowe, J. F. 2014, in IAU Symp., 293, ed. N. Haghighipour, 52

Endl, M., Robertson, P., Cochran, W. D., et al. 2012, ApJ, 759, 19

Fuhrmann, K. 1998, A\&A, 338, 161

Grevesse, N., \& Sauval, A. J. 1998, Space Sci. Rev., 85, 161

Guillot, T., \& Havel, M. 2011, A\&A, 527, A20

Guzik, J. A., Houdek, G., Chaplin, W. J., et al. 2011, ArXiv e-prints [arXiv: 1110.2120 ]

Holmberg, J., Nordström, B., \& Andersen, J. 2009, A\&A, 501, 941

Ibukiyama, A., \& Arimoto, N. 2002, A\&A, 394, 927

Iglesias, C. A., \& Rogers, F. J. 1996, ApJ, 464, 943

Jones, B. W., Sleep, P. N., \& Underwood, D. R. 2006, ApJ, 649, 1010

Jørgensen, B. R., \& Lindegren, L. 2005, A\&A, 436, 127

Kervella, P., Thévenin, F., Di Folco, E., \& Ségransan, D. 2004, A\&A, 426, 297

Lebreton, Y., \& Goupil, M. J. 2014, A\&A, 569, A21 
Lejeune, T., Cuisinier, F., \& Buser, R. 1997, VizieR Online Data Catalog: $\mathrm{J} / \mathrm{A}+\mathrm{AS} / 125 / 229$

Ligi, R., Mourard, D., Lagrange, A. M., et al. 2012a, A\&A, 545, A5

Ligi, R., Mourard, D., Lagrange, A.-M., Perraut, K., \& Tallon-Bosc, I. 2012b, in SPIE Conf. Ser., 8445

Ligi, R., Mourard, D., Nardetto, N., \& Clausse, J.-M. 2013, J. Astron. Instrum., 02,1340003

Ligi, R., Mourard, D., Lagrange, A. M., Perraut, K., \& Chiavassa, A. 2015, A\&A, 574, A69

Liu, Y.-J., Sato, B., Zhao, G., \& Ando, H. 2009, Res. Astron. Astrophys., 9, 1

Liu, Y., Sato, B., Takeda, Y., Ando, H., \& Zhao, G. 2010, PASJ, 62, 1071

Mamajek, E. E. 2009, in AIP Conf. Ser. 1158, eds. T. Usuda, M. Tamura, \& M. Ishii, 3

Marigo, P., \& Aringer, B. 2009, A\&A, 508, 1539

Maxted, P. F. L., Serenelli, A. M., \& Southworth, J. 2015, A\&A, 577, A90

Mayor, M., \& Queloz, D. 1995, Nature, 378, 355

Mayor, M., Udry, S., Naef, D., et al. 2004, A\&A, 415, 391

McArthur, B. E., Benedict, G. F., Barnes, R., et al. 2010, ApJ, 715, 1203

Mourard, D., Clausse, J. M., Marcotto, A., et al. 2009, A\&A, 508, 1073

Mourard, D., Challouf, M., Ligi, R., et al. 2012, in SPIE Conf. Ser., 8445

Mourard, D., Monnier, J. D., Meilland, A., et al. 2015, A\&A, 577, A51

Naef, D., Mayor, M., Korzennik, S. G., et al. 2003, A\&A, 410, 1051

Pont, F., \& Eyer, L. 2004, MNRAS, 351, 487
Rauer, H., \& Catala, C. 2012, in EGU General Assembly Conference Abstracts 14 , eds. A. Abbasi, \& N. Giesen, 7033

Ricker, G. R., Winn, J. N., Vanderspek, R., et al. 2014, in SPIE Conf. Ser., 9143, 20

Saffe, C., Gómez, M., \& Chavero, C. 2005, A\&A, 443, 609

Salasnich, B. 1999, Ph.D. Thesis, University of Padova

Sato, B., Toyota, E., Omiya, M., et al. 2008, PASJ, 60, 1317

Schultheis, M., Zasowski, G., Allende Prieto, C., et al. 2014, AJ, 148, 24

Sturmann, J., ten Brummelaar, T. A., Ridgway, S. T., et al. 2003, in SPIE Conf. Ser. 4838, ed. W. A. Traub, 1208

Tallon-Bosc, I., Tallon, M., Thiébaut, E., et al. 2008, in SPIE Conf. Ser., 7013

ten Brummelaar, T. A., McAlister, H. A., Ridgway, S. T., et al. 2005, ApJ, 628, 453

Torres, G., Andersen, J., \& Giménez, A. 2010, A\&ARv, 18, 67

Valenti, J. A., \& Fischer, D. A. 2005, ApJS, 159, 141

van Belle, G. T., \& von Braun, K. 2009, ApJ, 694, 1085

van Belle, G. T., van Belle, G., Creech-Eakman, M. J., et al. 2008, ApJS, 176, 276

van Leeuwen, F. 2007, A\&A, 474, 653

Vogt, S. S., Butler, R. P., Marcy, G. W., et al. 2005, ApJ, 632, 638

von Braun, K., Boyajian, T. S., ten Brummelaar, T. A., et al. 2011, ApJ, 740, 49

Winn, J. N., Matthews, J. M., Dawson, R. I., et al. 2011, ApJ, 737, L18

Wright, J. T., Marcy, G. W., Butler, R. P., \& Vogt, S. S. 2004, ApJS, 152, 261 
R. Ligi et al.: Radii, masses, and ages of 18 bright stars using interferometry and new estimations of exoplanetary parameters

\section{Appendix A: On the distributions of the random variables}

\section{A.1. Expected values of $L_{\star}$ and $T_{\text {eff }}$}

Let $F_{\text {bol }}$ be the random variable "bolometric flux of the star", $\theta$ the random variable "angular diameter of the star" and $d$ the random variable "distance to the star". We assume that they are all independent and Gaussian with respective means and standard deviations $\bar{F}_{\text {bol }}^{-}, \bar{\theta}, \bar{d}$, and $\sigma_{F_{\text {bol }}}, \sigma_{\theta}, \sigma_{d}$.

Let $L_{\star}=4 \pi F_{\text {bol }} d^{2}$ and $T_{\text {eff }}=\beta F_{\text {bol }}^{1 / 4} \theta^{-1 / 2}$ be two other random variables (with $\left.\beta=\left(4 / \sigma_{S B}\right)^{1 / 4}\right)$. We note that $d^{2}$, hence $L_{\star}$, do not have a Gaussian distribution. To compute the expected values of $L_{\star}$ and $T_{\text {eff }}$, let us note $F_{\text {bol }}=F_{\text {bol }}^{-}\left(1+F_{\text {bol }}\right), d=\bar{d}(1+$ $\tilde{d})$, and $\theta=\bar{\theta}(1+\tilde{\theta})$ so that $\tilde{F_{\text {bol }}}, \tilde{d}$ and $\tilde{\theta}$ are three independent Gaussian variables of mean 0 and respective standard deviations $\sigma_{F_{\mathrm{bol}}}=\sigma_{F_{\mathrm{bol}}} / F_{\mathrm{bol}}^{-}, \sigma_{\tilde{d}}=\sigma_{d} / \bar{d}$ and $\sigma_{\tilde{\theta}}=\sigma_{\theta} / \bar{\theta}$, assumed to be small:

$$
\begin{aligned}
E\left(L_{\star}\right)= & E\left(4 \pi F_{\mathrm{bol}} d^{2}\right)=E\left(4 \pi \overline{F_{\mathrm{bol}}} \bar{d}^{2}\left(1+\tilde{F_{\mathrm{bol}}}\right)(1+\tilde{d})^{2}\right) \\
= & 4 \pi \overline{F_{\mathrm{bol}}} \bar{d}^{2} E\left(1+\tilde{F_{\mathrm{bol}}}+2 \tilde{d}+2 \tilde{F_{\mathrm{bol}}} \tilde{d}+\tilde{d}^{2}+\tilde{F_{\mathrm{bol}}} \tilde{d}^{2}\right) \\
= & 4 \pi \overline{F_{\mathrm{bol}}} \bar{d}^{2}(1+\underbrace{E\left(\tilde{F_{\mathrm{bol}}}\right)}_{0}+2 \underbrace{E(\tilde{d})}_{0}+2 \underbrace{E\left(\tilde{F_{\mathrm{bol}}}\right)}_{0} \underbrace{E(\tilde{d})}_{0} \\
& +E\left(\tilde{d}^{2}\right)+\underbrace{E\left(\tilde{F_{\mathrm{bol}}}\right)}_{0} E\left(\tilde{d}^{2}\right)) \\
E\left(L_{\star}\right)= & 4 \pi \overline{F_{\mathrm{bol}}} \bar{d}^{2} \times\left(1+\sigma_{\tilde{d}^{2}}\right),
\end{aligned}
$$

where we used the linearity of the expected value and the fact that if $X$ and $Y$ are independent random variables, $E(X Y)=$ $E(X) E(Y)$.
One can show similarly that, to second order in $\sigma_{F_{\text {bol }}}$ and $\sigma_{\tilde{\theta}}$,

$E\left(T_{\mathrm{eff}}\right)=\beta{F_{\mathrm{bol}}^{-}}^{1 / 4} \bar{\theta}^{-1 / 2} \times\left(1+\frac{12\left(\sigma_{\tilde{\theta}}\right)^{2}-3\left(\sigma_{F_{\mathrm{bol}}}\right)^{2}}{32}\right)$.

\section{A.2. Covariance and correlation of $L_{\star}$ and $T_{\text {eff }}$}

Now that we have $E\left(L_{\star}\right)$ and $E\left(T_{\text {eff }}\right)$, we can compute their covariance: $\operatorname{Cov}\left(L_{\star}, T_{\text {eff }}\right)=E\left(\left[L_{\star}-E\left(L_{\star}\right)\right]\left[T_{\text {eff }}-E\left(T_{\text {eff }}\right)\right]\right)$. Using similar arguments to those above and expanding to second order again, one finds

$\operatorname{Cov}\left(L_{\star}, T_{\text {eff }}\right)=\frac{1}{4} \overline{L_{\star}} \overline{T_{\mathrm{eff}}} \sigma_{F_{\mathrm{bol}}}^{2}$,

noting $\overline{L_{\star}}=4 \pi \bar{F}_{\mathrm{bol}}^{-} \bar{d}^{2}<E\left(L_{\star}\right)$ and $\overline{T_{\mathrm{eff}}}=\beta{\overline{F_{\mathrm{bol}}}}^{1 / 4} \bar{\theta}^{-1 / 2} \neq$ $E\left(T_{\text {eff }}\right)$.

From this, we see that if $\sigma_{F_{\text {bol }}}=0$, i.e. in the ideal case where $F_{\text {bol }}$ were known exactly without uncertainty, then $L_{\star}$ and $T_{\text {eff }}$ are not correlated ${ }^{9}$. In the $L_{\star}-T_{\text {eff }}$ plane, the cloud of points drawn by our Monte-Carlo algorithm will be fitted by ellipses with vertical and horizontal axes. In contrast, then $\sigma_{L_{\star}}=\overline{L_{\star}} \sigma_{F_{\text {bol }}}$ and $\sigma_{T_{\text {eff }}}=\frac{1}{4} \overline{T_{\text {eff }}} \sigma_{F_{\text {bol }}}$ in the case where $\sigma_{d}=$ $\sigma_{\theta}=0$, so that in the end the correlation between $L_{\star}$ and $T_{\text {eff }}$ is $\operatorname{Corr}\left(L_{\star}, T_{\text {eff }}\right)=\operatorname{Cov}\left(L_{\star}, T_{\text {eff }}\right) / \sigma_{L_{\star}} \sigma_{T_{\text {eff }}}=1 ; L_{\star}$ and $T_{\text {eff }}$ are perfectly correlated and in the $L_{\star}-T_{\text {eff }}$ plane, the cloud of points will look like a thin diagonal line this time. From these two extreme cases, one can have a feeling of how important the correlation is by comparing $\sigma_{F_{\text {bol }}}$ with $\sigma_{\tilde{d}}$ and $\sigma_{\tilde{\theta}}$. In general, they are of the same order of magnitude, showing the importance of taking this correlation into account by using our algorithm instead of drawing $L_{\star}$ and $T_{\text {eff }}$ independently. 
Appendix B: Additional table and figures

Table B.1. Journal of observations.

\begin{tabular}{|c|c|c|c|c|c|c|}
\hline $\begin{array}{l}\text { HD } \\
\text { MJD }\end{array}$ & Seq. & $\begin{array}{c}B \\
(\mathrm{~m}) \\
\end{array}$ & $V^{2}$ & $e V^{2}$ & $\begin{array}{c}\lambda \\
(\mathrm{nm})\end{array}$ & $\begin{array}{c}\Delta \lambda \\
(\mathrm{nm})\end{array}$ \\
\hline \multicolumn{7}{|l|}{ HD 3651} \\
\hline 56163.5 & C1-T-C2 & 155.64 & 0.252 & 0.050 & 707 & 15 \\
\hline 56163.5 & & 155.64 & 0.279 & 0.050 & 735 & 15 \\
\hline 56164.5 & C1-T-C1 & 147.23 & 0.210 & 0.031 & 718 & 15 \\
\hline 56164.5 & & 210.43 & 0.008 & 0.050 & 718 & 15 \\
\hline 56495.5 & $\mathrm{~T}-\mathrm{C} 3$ & 140.08 & 0.273 & 0.035 & 700 & 20 \\
\hline 56495.5 & & 200.88 & 0.032 & 0.050 & 700 & 20 \\
\hline 56495.5 & & 140.08 & 0.347 & 0.067 & 710 & 20 \\
\hline 56495.5 & C3-T & 149.52 & 0.280 & 0.018 & 700 & 20 \\
\hline 56495.5 & & 149.52 & 0.221 & 0.033 & 710 & 20 \\
\hline 56495.5 & & 149.52 & 0.273 & 0.031 & 730 & 20 \\
\hline 56495.5 & $\mathrm{~T}-\mathrm{C} 3$ & 151.92 & 0.219 & 0.017 & 700 & 20 \\
\hline 56495.5 & & 216.60 & -0.028 & 0.045 & 700 & 20 \\
\hline 56495.5 & & 151.92 & 0.232 & 0.027 & 710 & 20 \\
\hline 56495.5 & & 151.92 & 0.296 & 0.038 & 730 & 20 \\
\hline 56495.5 & & 216.60 & -0.010 & 0.050 & 730 & 20 \\
\hline 56502.5 & C2-T-C1 & 33.18 & 0.893 & 0.187 & 700 & 20 \\
\hline 56502.5 & & 170.03 & 0.190 & 0.040 & 700 & 20 \\
\hline 56502.5 & & 202.70 & -0.010 & 0.065 & 700 & 20 \\
\hline 56502.5 & & 170.03 & 0.168 & 0.038 & 710 & 20 \\
\hline 56502.5 & & 202.70 & 0.014 & 0.072 & 710 & 20 \\
\hline 56502.5 & C1-T-C3 & 33.22 & 0.944 & 0.143 & 710 & 20 \\
\hline 56502.5 & & 171.41 & 0.084 & 0.083 & 710 & 20 \\
\hline 56502.5 & C3-T-C1 & 33.33 & 0.932 & 0.195 & 700 & 20 \\
\hline 56502.5 & & 172.99 & 0.119 & 0.027 & 700 & 20 \\
\hline 56502.5 & & 172.99 & 0.226 & 0.037 & 710 & 20 \\
\hline 56502.5 & & 205.75 & -0.001 & 0.061 & 710 & 20 \\
\hline \multicolumn{7}{|l|}{ HD 19994} \\
\hline 56197.5 & C4-T & 28.42 & 0.940 & 0.129 & 710 & 20 \\
\hline 56197.5 & & 150.12 & 0.090 & 0.114 & 710 & 20 \\
\hline 56197.5 & & 177.90 & 0.044 & 0.050 & 710 & 20 \\
\hline 56197.5 & & 28.42 & 0.890 & 0.143 & 730 & 20 \\
\hline 56227.5 & C5-T- C6 & 153.58 & 0.158 & 0.050 & 705 & 15 \\
\hline 56227.5 & & 219.09 & 0.034 & 0.050 & 705 & 15 \\
\hline 56227.5 & & 153.58 & 0.155 & 0.050 & 720 & 15 \\
\hline 56227.5 & & 219.09 & -0.013 & 0.050 & 720 & 15 \\
\hline 56227.5 & & 153.58 & 0.201 & 0.050 & 735 & 15 \\
\hline 56229.5 & C4-T-C6 & 29.66 & 0.852 & 0.050 & 643 & 15 \\
\hline 56229.5 & & 29.66 & 0.913 & 0.050 & 658 & 15 \\
\hline 56229.5 & & 29.66 & 0.934 & 0.050 & 672 & 13 \\
\hline 56529.5 & C6-T-C6 & 152.81 & 0.214 & 0.054 & 730 & 20 \\
\hline 56529.5 & & 215.65 & 0.013 & 0.075 & 730 & 20 \\
\hline 56534.5 & C6-T & 28.10 & 1.000 & 0.195 & 710 & 20 \\
\hline 56534.5 & C6-Т-C6 & 146.53 & 0.297 & 0.050 & 710 & 20 \\
\hline 56534.5 & & 151.20 & 0.197 & 0.050 & 710 & 20 \\
\hline 56534.5 & & 179.10 & 0.069 & 0.067 & 710 & 20 \\
\hline 56533.5 & & 142.17 & 0.304 & 0.050 & 730 & 25 \\
\hline 56533.5 & & 169.32 & 0.054 & 0.053 & 730 & 25 \\
\hline 56534.5 & & 146.53 & 0.242 & 0.050 & 730 & 25 \\
\hline 56534.5 & & 151.20 & 0.246 & 0.050 & 730 & 25 \\
\hline 56534.5 & & 179.10 & 0.100 & 0.050 & 730 & 25 \\
\hline 56533.5 & & 27.82 & 0.896 & 0.079 & 748 & 25 \\
\hline 56533.5 & & 169.32 & 0.123 & 0.053 & 748 & 25 \\
\hline 56534.5 & & 151.20 & 0.326 & 0.050 & 748 & 25 \\
\hline 56534.5 & & 179.10 & 0.083 & 0.050 & 748 & 25 \\
\hline
\end{tabular}


R. Ligi et al.: Radii, masses, and ages of 18 bright stars using interferometry and new estimations of exoplanetary parameters

Table B.1. continued.

\begin{tabular}{|c|c|c|c|c|c|c|}
\hline $\begin{array}{l}\text { HD } \\
\text { MJD }\end{array}$ & Seq. & $\begin{array}{l}B \\
(\mathrm{~m})\end{array}$ & $V^{2}$ & $e V^{2}$ & $\begin{array}{l}\lambda \\
(\mathrm{nm})\end{array}$ & $\begin{array}{l}\Delta \lambda \\
(\mathrm{nm})\end{array}$ \\
\hline \multicolumn{7}{|l|}{ HD 75 732} \\
\hline 56034.5 & C7-T-C7 & 56.96 & 0.843 & 0.050 & 708 & 15 \\
\hline 56034.5 & & 276.00 & 0.021 & 0.056 & 708 & 15 \\
\hline 56034.5 & & 276.05 & -0.029 & 0.050 & 720 & 16 \\
\hline 56034.5 & & 56.96 & 0.857 & 0.050 & 733 & 15 \\
\hline 56034.5 & & 276.00 & -0.063 & 0.093 & 733 & 15 \\
\hline 56033.5 & C7-T-C7 & 56.33 & 0.853 & 0.050 & 720 & 16 \\
\hline 56033.5 & & 56.33 & 0.858 & 0.050 & 733 & 15 \\
\hline 56033.5 & & 244.57 & 0.044 & 0.052 & 733 & 15 \\
\hline 55887.5 & C8-T-C8 & 174.23 & 0.151 & 0.050 & 708 & 15 \\
\hline 55887.5 & & 174.70 & 0.204 & 0.069 & 708 & 15 \\
\hline 55888.5 & C8-T & 107.87 & 0.525 & 0.095 & 708 & 15 \\
\hline 55887.5 & C8-T-C8 & 174.23 & 0.158 & 0.050 & 720 & 16 \\
\hline 55887.5 & & 174.70 & 0.115 & 0.061 & 720 & 16 \\
\hline 55888.5 & C8-T & 107.87 & 0.459 & 0.050 & 720 & 16 \\
\hline 55888.5 & & 177.20 & 0.131 & 0.086 & 720 & 16 \\
\hline 55887.5 & C8-T-C8 & 99.01 & 0.595 & 0.078 & 733 & 15 \\
\hline 55887.5 & & 174.69 & 0.079 & 0.073 & 733 & 15 \\
\hline 55887.5 & & 238.14 & 0.050 & 0.077 & 733 & 15 \\
\hline 55888.5 & C8-T & 107.89 & 0.584 & 0.097 & 733 & 15 \\
\hline 55888.5 & & 177.22 & 0.219 & 0.086 & 733 & 15 \\
\hline \multicolumn{7}{|l|}{ HD 167042} \\
\hline 56190.5 & C9-T & 64.77 & 0.579 & 0.050 & 710 & 20 \\
\hline 56191.5 & С9-Т-С9 & 65.48 & 0.639 & 0.050 & 710 & 20 \\
\hline 56190.5 & C9-T & 64.78 & 0.420 & 0.033 & 539.5 & 15 \\
\hline 56190.5 & C9-T & 151.34 & 0.081 & 0.125 & 539.5 & 15 \\
\hline 56190.5 & C9-T & 64.77 & 0.607 & 0.050 & 730 & 20 \\
\hline 56190.5 & C9-T & 215.89 & -0.057 & 0.115 & 730 & 20 \\
\hline 56191.5 & C9-Т-C9 & 65.48 & 0.617 & 0.050 & 730 & 20 \\
\hline 56191.5 & C9-Т-С9 & 219.74 & -0.064 & 0.138 & 730 & 20 \\
\hline 56191.5 & С9-Т-С9 & 65.48 & 0.405 & 0.151 & 552.5 & 15 \\
\hline 56190.5 & C9-T & 64.78 & 0.410 & 0.050 & 539.5 & 15 \\
\hline 56190.5 & C9-T & 151.34 & 0.086 & 0.093 & 539.5 & 15 \\
\hline 56190.5 & C9-T & 64.77 & 0.639 & 0.050 & 747.5 & 25 \\
\hline 56190.5 & C9-T & 215.89 & 0.097 & 0.135 & 747.5 & 25 \\
\hline 56501.5 & C9-Т-C9 & 64.81 & 0.590 & 0.050 & 710 & 20 \\
\hline 56501.5 & C9-Т-C9 & 64.81 & 0.553 & 0.083 & 727.5 & 25 \\
\hline \multicolumn{7}{|l|}{ HD 173416} \\
\hline 56533.5 & C10-T-C10 & 65.58 & 0.714 & 0.072 & 710 & 20 \\
\hline 56533.5 & & 155.80 & 0.073 & 0.070 & 710 & 20 \\
\hline 56533.5 & & 221.07 & 0.019 & 0.082 & 710 & 20 \\
\hline 56533.5 & C10-T-C10 & 64.17 & 0.586 & 0.050 & 710 & 20 \\
\hline 56533.5 & & 152.36 & 0.020 & 0.083 & 710 & 20 \\
\hline 56533.5 & C10-T-C10 & 65.58 & 0.596 & 0.082 & 730 & 20 \\
\hline 56533.5 & & 155.80 & 0.086 & 0.050 & 730 & 20 \\
\hline 56533.5 & & 221.07 & -0.044 & 0.050 & 730 & 20 \\
\hline 56533.5 & C10-T-C10 & 64.17 & 0.698 & 0.089 & 730 & 20 \\
\hline 56533.5 & & 152.36 & 0.046 & 0.050 & 730 & 20 \\
\hline \multicolumn{7}{|l|}{ HD 190360} \\
\hline 56496.3 & C10-T-C10 & 175.50 & 0.277 & 0.034 & 700 & 20 \\
\hline 56496.3 & & 33.97 & 0.917 & 0.040 & 710 & 20 \\
\hline 56496.3 & & 175.50 & 0.241 & 0.050 & 710 & 20 \\
\hline 56496.3 & & 175.50 & 0.291 & 0.028 & 727.5 & 25 \\
\hline 56496.3 & C10-T-C10 & 33.98 & 0.879 & 0.062 & 700 & 20 \\
\hline 56496.3 & & 176.00 & 0.172 & 0.052 & 700 & 20 \\
\hline 56496.3 & & 33.98 & 0.927 & 0.074 & 710 & 20 \\
\hline 56501.4 & C11-T-C12 & 176.13 & 0.214 & 0.023 & 700 & 20 \\
\hline 56501.4 & & 243.67 & -0.040 & 0.105 & 700 & 20 \\
\hline 56501.4 & & 176.13 & 0.226 & 0.016 & 710 & 20 \\
\hline 56501.4 & & 176.13 & 0.163 & 0.039 & 730 & 20 \\
\hline 56501.4 & C12-T-C12 & 174.19 & 0.262 & 0.020 & 700 & 20 \\
\hline 56501.4 & & 174.11 & 0.197 & 0.058 & 710 & 20 \\
\hline 56501.4 & & 174.11 & 0.281 & 0.026 & 730 & 20 \\
\hline
\end{tabular}


Table B.1. continued.

\begin{tabular}{|c|c|c|c|c|c|c|}
\hline $\begin{array}{l}\text { HD } \\
\text { MJD }\end{array}$ & Seq. & $\begin{array}{l}B \\
(\mathrm{~m})\end{array}$ & $V^{2}$ & $e V^{2}$ & $\begin{array}{l}\lambda \\
(\mathrm{nm})\end{array}$ & $\begin{array}{l}\Delta \lambda \\
(\mathrm{nm})\end{array}$ \\
\hline 56123.3 & C11-T-C11 & 218.43 & 0.124 & 0.060 & 730 & 20 \\
\hline 56124.3 & C11-T & 221.80 & 0.050 & 0.070 & 730 & 20 \\
\hline 56130.4 & $\mathrm{C} 11-\mathrm{T}$ & 152.66 & 0.410 & 0.098 & 707.5 & 15 \\
\hline 56130.4 & & 152.73 & 0.416 & 0.110 & 730 & 20 \\
\hline 56130.4 & & 248.07 & -0.001 & 0.094 & 730 & 20 \\
\hline \multicolumn{7}{|c|}{ HD 217014} \\
\hline 56164.5 & C12-T-C1 & 64.55 & 0.870 & 0.035 & 708 & 15 \\
\hline 56164.5 & & 64.55 & 0.842 & 0.027 & 720 & 20 \\
\hline 56164.5 & & 64.55 & 0.886 & 0.024 & 733 & 15 \\
\hline 56164.5 & & 150.70 & 0.270 & 0.025 & 720 & 20 \\
\hline 56164.5 & & 150.70 & 0.300 & 0.047 & 733 & 15 \\
\hline 56164.5 & & 215.02 & 0.009 & 0.037 & 720 & 20 \\
\hline 56164.5 & & 215.02 & 0.049 & 0.064 & 733 & 15 \\
\hline 56495.5 & C12-T-C12 & 168.42 & 0.189 & 0.039 & 700 & 20 \\
\hline 56495.5 & & 168.42 & 0.289 & 0.022 & 710 & 20 \\
\hline 56495.5 & & 168.42 & 0.236 & 0.050 & 730 & 20 \\
\hline 56495.5 & C12-T-C12 & 168.07 & 0.257 & 0.036 & 700 & 20 \\
\hline 56495.5 & & 168.07 & 0.276 & 0.058 & 710 & 20 \\
\hline 56495.5 & & 168.07 & 0.281 & 0.046 & 730 & 20 \\
\hline \multicolumn{7}{|l|}{ HD 1367} \\
\hline 56196.5 & C4-T-C4 & 63.25 & 0.740 & 0.050 & 727 & 30 \\
\hline 56196.5 & & 153.10 & 0.190 & 0.050 & 727 & 30 \\
\hline 56226.5 & C4-T-C5 & 65.10 & 0.721 & 0.051 & 705 & 15 \\
\hline 56226.5 & & 155.92 & 0.228 & 0.050 & 705 & 15 \\
\hline 56226.5 & & 220.87 & 0.000 & 0.073 & 705 & 15 \\
\hline 56227.5 & C4-T-C5 & 154.51 & 0.204 & 0.050 & 705 & 15 \\
\hline 56227.5 & & 154.51 & 0.209 & 0.050 & 720 & 15 \\
\hline 56227.5 & & 218.42 & 0.051 & 0.050 & 720 & 15 \\
\hline 56227.5 & & 154.51 & 0.228 & 0.050 & 735 & 15 \\
\hline 56227.5 & & 218.42 & 0.000 & 0.050 & 735 & 15 \\
\hline \multicolumn{7}{|l|}{ HD 1671} \\
\hline 56198.5 & $\mathrm{~T}-\mathrm{C} 13$ & 210.97 & 0.111 & 0.050 & 710 & 20 \\
\hline 56198.5 & & 271.69 & 0.007 & 0.050 & 730 & 20 \\
\hline 56198.5 & C13-T-C13 & 210.97 & 0.167 & 0.050 & 710 & 20 \\
\hline 56198.5 & & 276.04 & -0.015 & 0.050 & 710 & 20 \\
\hline 56198.5 & & 210.97 & 0.120 & 0.050 & 730 & 20 \\
\hline 56198.5 & & 276.04 & 0.044 & 0.050 & 730 & 20 \\
\hline 56528.5 & C13-T-C14 & 150.46 & 0.426 & 0.050 & 710 & 20 \\
\hline 56528.5 & & 213.65 & 0.093 & 0.050 & 710 & 20 \\
\hline 56528.5 & & 150.46 & 0.436 & 0.050 & 730 & 20 \\
\hline 56528.5 & & 63.53 & 0.873 & 0.050 & 748 & 25 \\
\hline 56529.5 & C14-T-C14 & 177.42 & 0.259 & 0.050 & 710 & 20 \\
\hline 56529.5 & & 210.90 & 0.131 & 0.050 & 710 & 20 \\
\hline 56529.5 & & 177.42 & 0.351 & 0.050 & 730 & 20 \\
\hline 56533.5 & & 210.95 & 0.091 & 0.050 & 710 & 20 \\
\hline 56533.5 & C15-T-C15 & 138.25 & 0.466 & 0.050 & 710 & 20 \\
\hline 56533.5 & & 197.37 & 0.120 & 0.050 & 710 & 20 \\
\hline 56533.5 & & 138.25 & 0.488 & 0.050 & 730 & 20 \\
\hline 56533.5 & & 197.37 & 0.191 & 0.050 & 730 & 20 \\
\hline 56533.5 & & 59.44 & 0.888 & 0.050 & 748 & 25 \\
\hline 56533.5 & & 138.25 & 0.451 & 0.050 & 748 & 25 \\
\hline 56533.5 & C13-T-C13 & 177.44 & 0.273 & 0.050 & 730 & 20 \\
\hline 56533.5 & & 210.95 & 0.097 & 0.050 & 730 & 20 \\
\hline 56533.5 & & 177.44 & 0.290 & 0.050 & 748 & 25 \\
\hline 56533.5 & & 210.95 & 0.103 & 0.050 & 748 & 25 \\
\hline \multicolumn{7}{|c|}{ HD 154633} \\
\hline 55722.5 & C16-T-C16 & 202.47 & 0.035 & 0.050 & 715 & 20 \\
\hline 55722.5 & & 202.47 & 0.079 & 0.050 & 735 & 20 \\
\hline 55721.5 & C16-C17-T-C16 & 105.13 & 0.425 & 0.066 & 711 & 18 \\
\hline 55721.5 & & 140.77 & 0.220 & 0.050 & 711 & 18 \\
\hline 55721.5 & & 233.81 & 0.056 & 0.052 & 711 & 18 \\
\hline 55721.5 & C16-C17-T-C16 & 105.13 & 0.437 & 0.050 & 735 & 20 \\
\hline
\end{tabular}


R. Ligi et al.: Radii, masses, and ages of 18 bright stars using interferometry and new estimations of exoplanetary parameters

Table B.1. continued.

\begin{tabular}{|c|c|c|c|c|c|c|}
\hline $\begin{array}{l}\text { HD } \\
\text { MJD }\end{array}$ & Seq. & $\begin{array}{l}B \\
(\mathrm{~m})\end{array}$ & $V^{2}$ & $e V^{2}$ & $\begin{array}{l}\lambda \\
(\mathrm{nm})\end{array}$ & $\begin{array}{l}\Delta \lambda \\
(\mathrm{nm})\end{array}$ \\
\hline 55721.5 & & 140.77 & 0.243 & 0.050 & 735 & 20 \\
\hline 55721.5 & C16-T-C16 & 107.74 & 0.458 & 0.050 & 711 & 18 \\
\hline 55721.5 & & 247.24 & -0.003 & 0.050 & 711 & 18 \\
\hline 55721.5 & C16-T-C16 & 107.74 & 0.466 & 0.050 & 735 & 20 \\
\hline 55721.5 & & 151.27 & 0.199 & 0.050 & 735 & 20 \\
\hline \multicolumn{7}{|c|}{ HD 161178} \\
\hline 56165.5 & C16-T-C16 & 65.26 & 0.582 & 0.050 & 710 & 20 \\
\hline 56165.5 & & 155.92 & 0.010 & 0.050 & 710 & 20 \\
\hline 56165.5 & & 220.96 & -0.032 & 0.050 & 710 & 20 \\
\hline 56165.5 & C16-T-C16 & 65.26 & 0.600 & 0.050 & 730 & 20 \\
\hline 56168.5 & C16-T-C18 & 101.66 & 0.391 & 0.050 & 710 & 20 \\
\hline 56168.5 & & 101.66 & 0.474 & 0.050 & 730 & 20 \\
\hline 56168.5 & & 155.97 & 0.042 & 0.050 & 730 & 20 \\
\hline \multicolumn{7}{|c|}{ HD 168151} \\
\hline 55722.5 & C16-T-C16 & 105.05 & 0.672 & 0.080 & 735 & 20 \\
\hline 55722.5 & & 199.04 & 0.141 & 0.050 & 735 & 20 \\
\hline 55722.5 & & 288.56 & -0.043 & 0.063 & 735 & 20 \\
\hline 55722.5 & C16-T-C16 & 107.72 & 0.522 & 0.067 & 735 & 20 \\
\hline 55722.5 & & 214.20 & 0.110 & 0.050 & 735 & 20 \\
\hline 55722.5 & & 306.93 & -0.054 & 0.065 & 735 & 20 \\
\hline 55721.5 & C16-T-C17-C16 & 104.07 & 0.529 & 0.050 & 735 & 20 \\
\hline 55721.5 & & 137.01 & 0.410 & 0.055 & 735 & 20 \\
\hline 55721.5 & & 228.95 & 0.048 & 0.050 & 735 & 20 \\
\hline 55721.5 & C16-T-C17-C16 & 107.36 & 0.579 & 0.051 & 735 & 20 \\
\hline 55721.5 & & 149.40 & 0.351 & 0.057 & 735 & 20 \\
\hline 55721.5 & & 244.86 & 0.070 & 0.050 & 735 & 20 \\
\hline \multicolumn{7}{|c|}{ HD 209369} \\
\hline 55767.4 & T-C19 & 106.06 & 0.592 & 0.05 & 710 & 16 \\
\hline 55767.4 & & 144.00 & 0.272 & 0.05 & 710 & 16 \\
\hline 55774.3 & C19-T-C19 & 107.47 & 0.597 & 0.05 & 645.5 & 15 \\
\hline 55774.3 & & 144.82 & 0.416 & 0.05 & 645.5 & 15 \\
\hline 55802.5 & C19-T & 103.21 & 0.732 & 0.05 & 737.5 & 15 \\
\hline 55802.5 & & 155.75 & 0.371 & 0.05 & 737.5 & 15 \\
\hline 55802.5 & С20-Т-C19 & 102.08 & 0.660 & 0.05 & 737.5 & 15 \\
\hline 55802.5 & & 155.77 & 0.341 & 0.05 & 737.5 & 15 \\
\hline 55803.4 & C19-T-C20 & 104.83 & 0.620 & 0.05 & 737.5 & 15 \\
\hline 55803.4 & & 155.41 & 0.293 & 0.05 & 737.5 & 15 \\
\hline 55803.4 & & 251.17 & 0.079 & 0.05 & 737.5 & 15 \\
\hline \multicolumn{7}{|c|}{ HD 218560} \\
\hline 55768.5 & C19-T-C19 & 64.18 & 0.704 & 0.060 & 710 & 16 \\
\hline 55768.5 & & 153.18 & 0.076 & 0.050 & 710 & 16 \\
\hline 55768.5 & & 217.05 & -0.004 & 0.050 & 710 & 16 \\
\hline 55768.5 & C19-T & 65.48 & 0.768 & 0.054 & 710 & 16 \\
\hline 55768.5 & & 155.86 & 0.109 & 0.050 & 710 & 16 \\
\hline 55802.5 & C19-T-C19 & 107.79 & 0.283 & 0.050 & 710 & 16 \\
\hline 55802.5 & & 151.98 & 0.082 & 0.050 & 710 & 16 \\
\hline 55802.5 & & 247.99 & 0.049 & 0.060 & 710 & 16 \\
\hline 55802.5 & C19-T-C19 & 107.79 & 0.274 & 0.050 & 710 & 16 \\
\hline 55802.5 & & 151.98 & 0.076 & 0.050 & 710 & 16 \\
\hline 55802.5 & & 247.99 & 0.046 & 0.057 & 710 & 16 \\
\hline
\end{tabular}



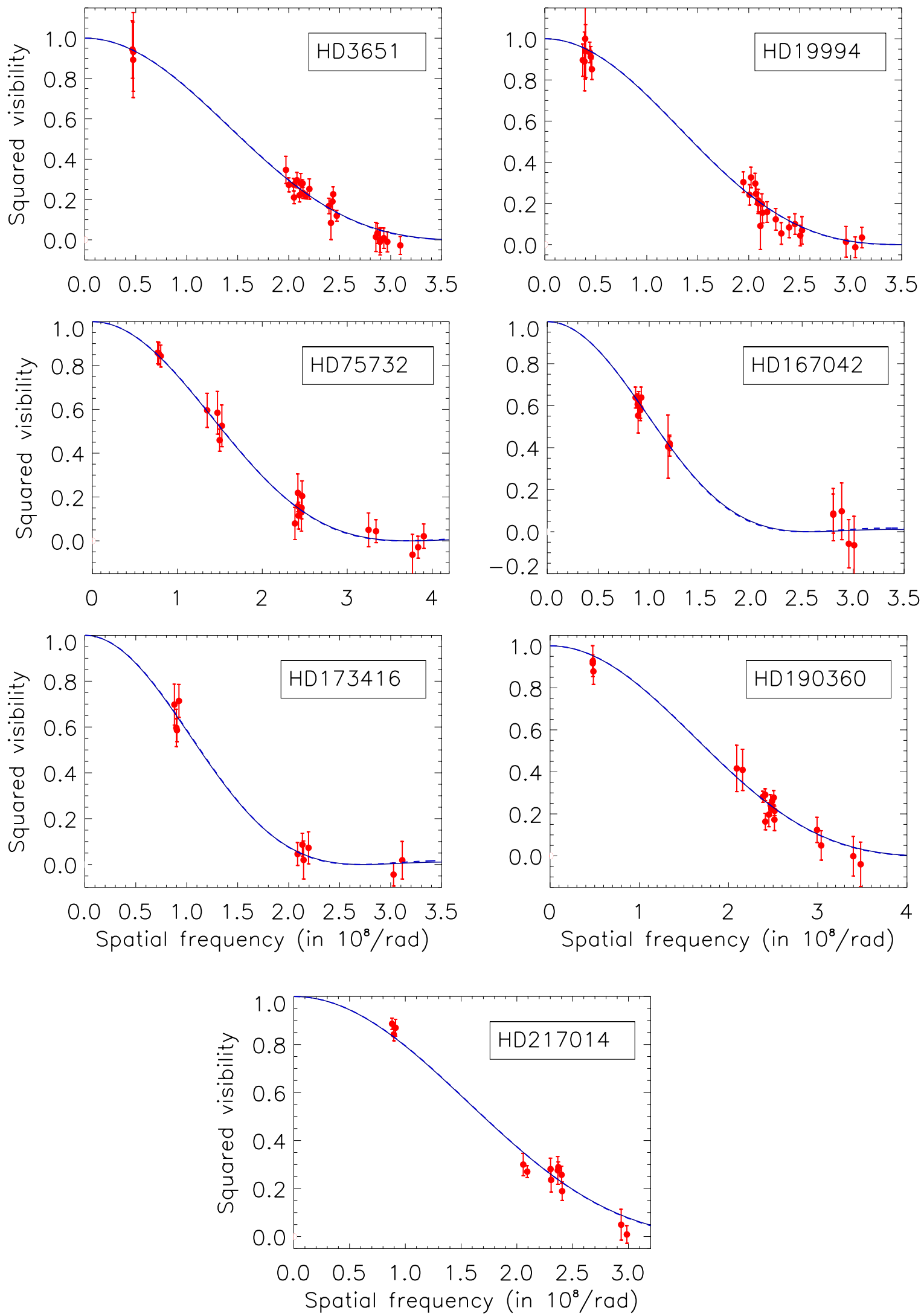

Fig. B.1. Squared visibilities of exoplanet host stars. The solid line represents the model of LD diameter and the dashed line the UD diameter (see Sect. 2). 
R. Ligi et al.: Radii, masses, and ages of 18 bright stars using interferometry and new estimations of exoplanetary parameters
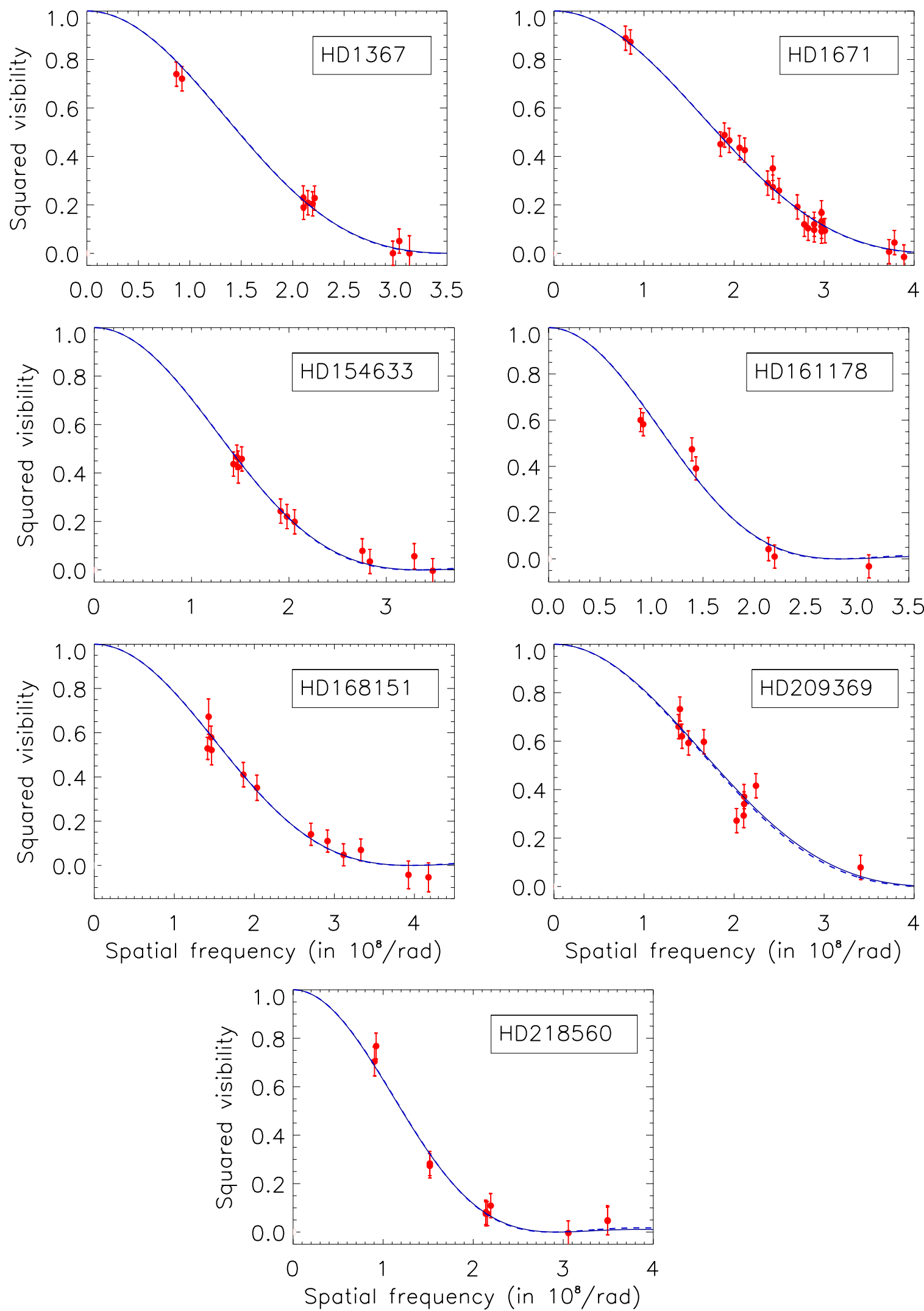

Fig. B.2. Squared visibilities of single stars. The solid line represents the model of LD diameter and the dashed line the UD diameter (see Sect. 2). 

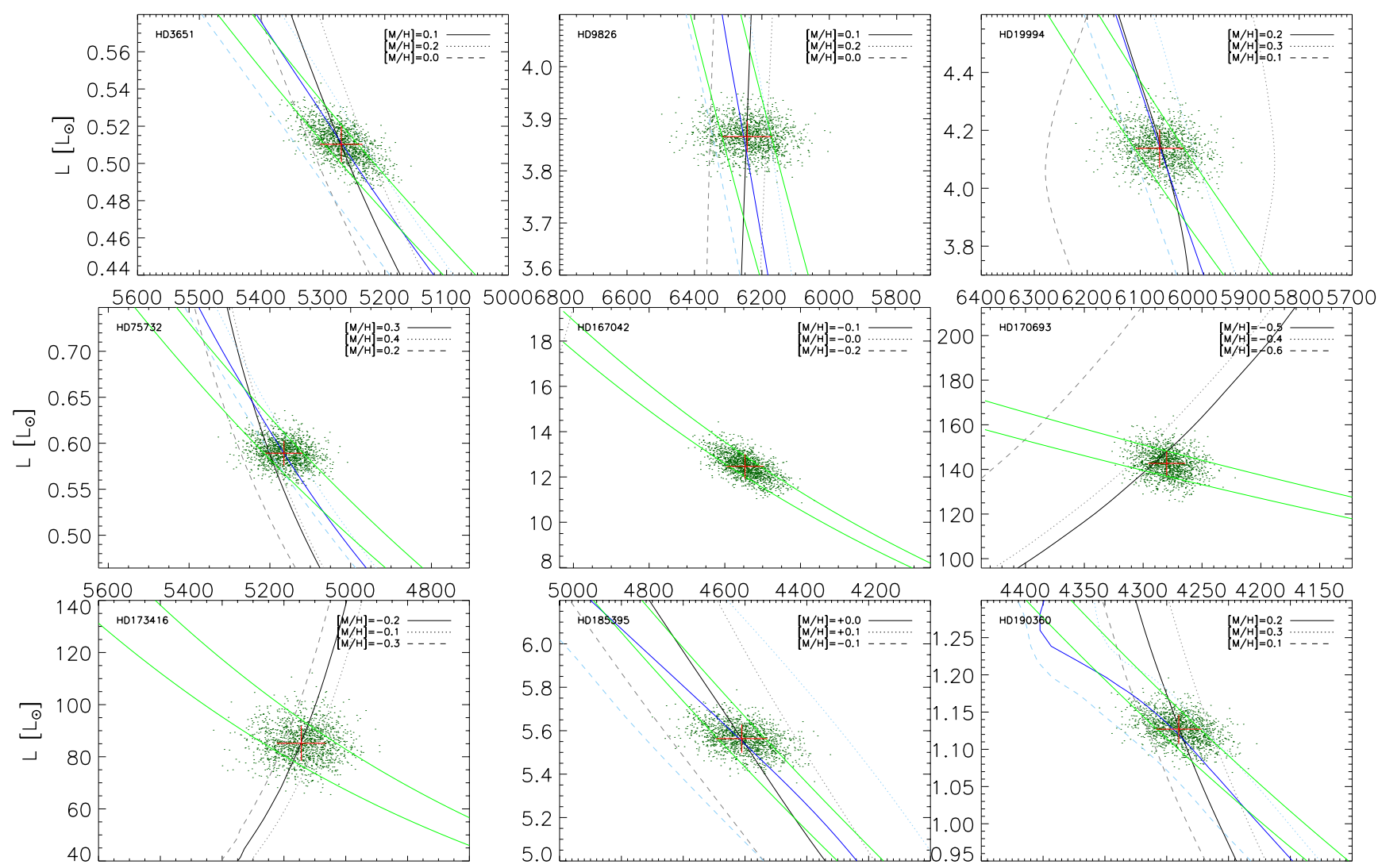

5200500048004600440042004000
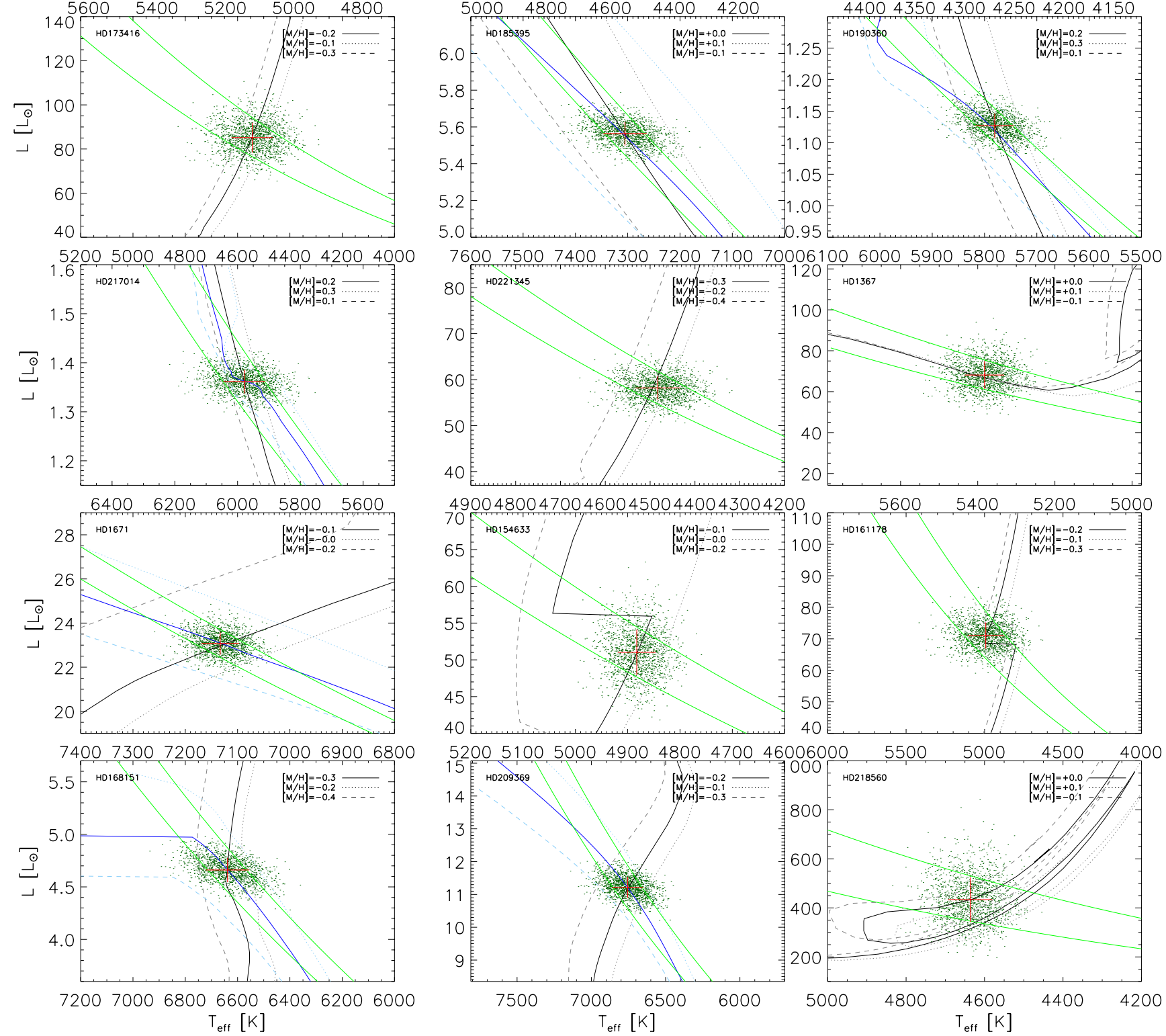

Fig. B.3. Isochrones from PARSEC models. The clouds of points represent the MC distribution. The black lines are for old solutions and the blue lines for young solutions. The isochrones of the stellar age at metallicity of \pm 0.1 dex are plotted in lighter colors than the reference (dotted and dashed lines). The solid light green lines represent the contraints of the radius at $1 \sigma$ (see Sect. 4). 
R. Ligi et al.: Radii, masses, and ages of 18 bright stars using interferometry and new estimations of exoplanetary parameters
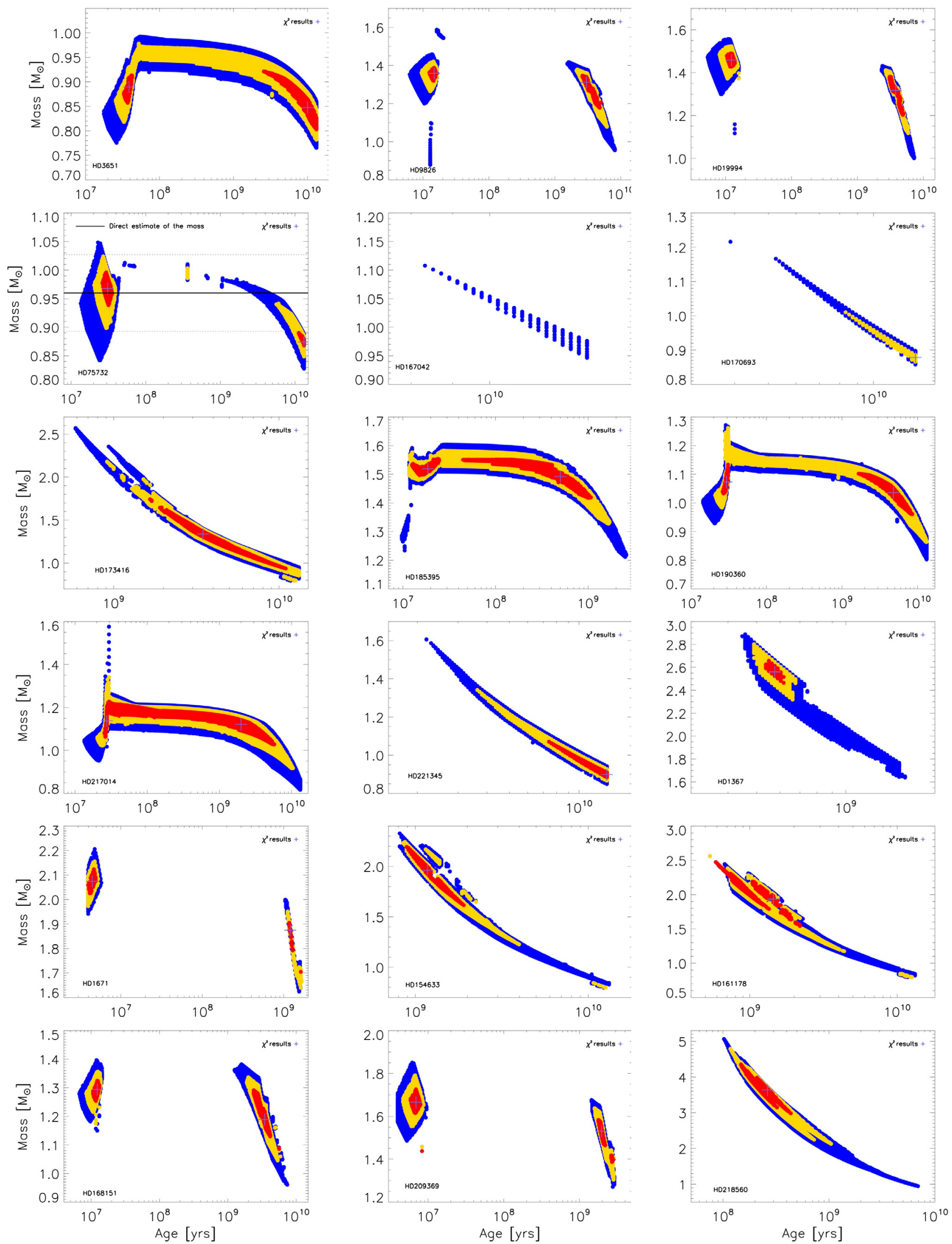

Fig. B.4. Approximate contour maps of the likelihood $\mathcal{L}$ as a function of the stellar masses and ages. Values within 1,2, and 3 of each term of Eq. (11) appear in red, yellow, and blue, respectively. Considering a flat prior (which is reasonable), these maps show the joint PDF of $M_{\star}$ and the age. The best fit old and young (if any) solutions are represented by crosses. The solid horizontal line in the case of HD 75732 corresponds to an independent estimate of the mass, and the dashed horizontal lines correspond to the errobars on $M_{\star}$ (see Sect. 4.3). 